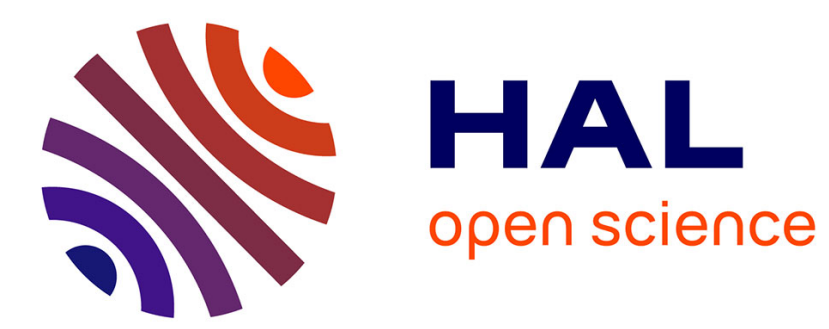

\title{
A model of social welfare improving transfers
}

Brice Magdalou

\section{To cite this version:}

| Brice Magdalou. A model of social welfare improving transfers. 2018. hal-01975452

\section{HAL Id: hal-01975452 \\ https://hal.science/hal-01975452}

Preprint submitted on 9 Jan 2019

HAL is a multi-disciplinary open access archive for the deposit and dissemination of scientific research documents, whether they are published or not. The documents may come from teaching and research institutions in France or abroad, or from public or private research centers.
L'archive ouverte pluridisciplinaire HAL, est destinée au dépôt et à la diffusion de documents scientifiques de niveau recherche, publiés ou non, émanant des établissements d'enseignement et de recherche français ou étrangers, des laboratoires publics ou privés. 


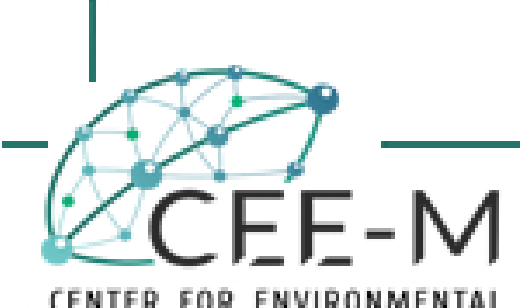

CENTER FOR ENVIRONMENTAL ECONOMICS - MONTPELLIER

\title{
A model of social welfare improving transfers
}

\author{
Brice Magdalou
}

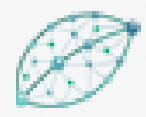

CEE-M Working Paper 2018-13 


\title{
A model of social welfare improving transfers *
}

\author{
Brice Magdalou ${ }^{\dagger}$ \\ CEE-M Working Paper 2018-13
}

\begin{abstract}
We establish an equivalence theorem between (i) dominance of one society by another, according to a finite sequence of social welfare improving transfers and (ii) dominance according to a class of social welfare functions, in the following framework: individual outcomes are multidimensional but finitely divisible in each dimension, a distribution simply counts the number of individuals having each possible outcome, and the considered set of transfers has the structure of a discrete cone. This framework encompasses most of the social welfare improving transfers investigated in the literature such as, for instance, Pigou-Dalton progressive transfers. As by-products, our model sheds new light on some surprising results in the literature on social deprivation, and provides new arguments on the key role of the expected utility model in decision-making under risk.
\end{abstract}

JEL Classification Numbers: C02, D63, D81.

Keywords: social welfare, inequality, welfare-improving transfers, stochastic dominance.

* This paper forms part of the two research projects RediPref (Contract ANR-15-CE26-0004) and OrdIneq (Contract ANR-16-CE41-0005) of the French National Agency for Research (ANR) whose financial support is gratefully acknowledged. It has also been supported by LabEx Entrepreneurship (Contract ANR-10-LABX-11-01).

$\dagger$ CEE-M, Univ. Montpellier, CNRS, INRA, SupAgro, Montpellier, France.

E-mail: brice.magdalou@umontpellier.fr. 


\section{Introduction}

The main challenge of any model of social welfare assessment (and related concepts such as inequality, poverty, equality of opportunity, social mobility, ...) is to define a transformation of the individual outcomes, or endowments, the social planner considers as unambiguously social welfare improving. The second difficulty is to provide measurement tools, consistent with the social welfare views captured by such transfers. Among the possible approaches to compare outcome distributions, the strategy which consists in providing criteria (called quasi-orderings) conclusive only when one distribution is obtained from the other by a sequence of such transfers, is probably the less questionnable one from a normative perspective: The ranking of the distributions is in that case crystal clear, because it is inextricably linked to our own definition of what we call 'social welfare improvement'.

In this paper, we investigate these two related issues at an abstract and broad level. We do not consider 'specific' social welfare improving transfers, as usually done in the literature, but we define a general set of transfers on the basis of the minimal properties such a set could satisfy. As illustrated in the last four sections our approach generalizes, with a few exceptions, all the usual transfers considered in the literature. It follows that some important results in this literature appear to be simple corollaries of the main theorem we present here. More importantly, this theorem opens new research avenues because it can be used in various contexts, with new and original notions of social welfare improving transfers. It can also be applied in other economic fields such that, for instance, the theory of decision under risk.

The most prominent example is the measurement model built around the PigouDalton principle of transfers, whereby a mean-preserving income transfer from an individual to a poorer one is still deemed inequality-reducing. In this context, the Hardy et al. (1952)'s theorem (HLP hereafter) can be regarded as a cornerstone for comparing income distributions. ${ }^{1}$ For any equal mean income distributions $x$ and $x^{\prime}$, by letting $\mathbb{E}$ be the expectation operator and $u$ a utility function, they have established the equivalence between the following three statements:

(a) $x$ is obtained from $x^{\prime}$ by means of a finite sequence of Pigou-Dalton transfers,

(b) $\mathbb{E}[u(x)] \geq \mathbb{E}\left[u\left(x^{\prime}\right)\right]$ for all $u$ concave,

(c) The Lorenz curve of $x$ lies nowhere below that of $x^{\prime}$.

The first statement describes a situation where one distribution is less unequal than another, according to the principle of transfers views on inequality. The second statement describes a unanimous ranking between the distributions, among all the utilitarian social planners endowed with a concave utility function. The last statement is an empirically implementable criterion, with a conclusive ranking of the distributions only when the first one is unambiguously less unequal than the other.

1 This result has been popularized in normative economics by Kolm (1969), Atkinson (1970), Dasgupta et al. (1973), Sen (1973) and Fields and Fei (1978). 
Our main objective is to obtain a comparable result, in a very general framework. Individual outcomes are assumed to be multidimensional, in order to capture the main dimensions characterizing welfare. By considering without loss of generality societies of equal size, a distribution simply counts the number of individuals having each possible outcome. Then we assume that each outcome dimension is finitely divisible. We emphasize that this discrete framework is relevant for any variable for which data are available in practice, be it cardinal or ordinal. Take income as an example, a cardinal variable which is, in addition, usually addressed by the theory as a continuous variable. In empirical data, it turns out that income is always provided on a discrete scale (euro cents for instance). This discrete framework is also well-suited for ordinal variables (health, education, ....), as they are usually defined on an ordered categorical scale. It is therefore possible to combine cardinal and ordinal dimensions in a unified approach. ${ }^{2}$

Within this framework, this paper provides a generalization of the equivalence between statements (a) and (b) of the HLP theorem (which are, actually, two quasiorderings). We do not consider specific transfers, but an 'abstract set of transfers' endowed with the following two properties: a transfer can be written as the difference between two outcome distributions and, if a transfer is assumed to be welfare improving, the reverse transfer is welfare decreasing. Then a 'sequence of transfers' is described as a linear combination of transfers. It follows that such a sequence has the structure of a 'discrete cone'. Hence, our general statement (a) is nothing else than a 'quasi-ordering induced by a discrete cone'. Apart from the generality of our framework this paper is the first, to the best of our knowledge, which directly investigates the equivalence between statements of types (a) and (b) in the literature of social welfare measurement (in a broad sense). Indeed usual approaches establish, separately, the equivalences between (a) and (c), and between (b) and (c).

The equivalence between statements (a) and (b) above can be compared to the equivalence, also well-known, between the following two statements:

(d) For all distributions $x$ and $x^{\prime}, x$ being obtained from $x^{\prime}$ by means of a finite sequence of Pigou-Dalton transfers implies $\mathbb{E}[u(x)] \geq \mathbb{E}\left[u\left(x^{\prime}\right)\right]$,

(e) $u$ is concave.

One can easily see that the implications '(a) implies (b)' and '(e) implies (d)' can be proved with identical arguments. Hence they provide the same information, which is not the case for the reverse implications. The implication '(b) implies (a)' establishes that the unanimity of ranking among all the utilitarian social planners, endowed with a concave utility function, is sufficient to ensure that the first distribution is obtained from the second one by means of a sequence of Pigou-Dalton transfers, whereas the implication '(d) implies (e)' establishes that the concavity of the social planner's utility

2 Focusing on the distinction between 'continuous' and 'discrete', a continuous scale can always be considered as the limit point of a 'very narrow' discrete scale. For instance, Gravel et al. (2014) provide an HLP theorem for a discrete ordinal variable, by substituting the so-called 'Hammond transfers' for the Pigou-Dalton transfers. They establish that the implementation criterion they obtain converges, when the discrete scale is 'refined', to the implementation criterion that can be obtained for a continuous ordinal variable, as identified by Gravel et al. (2019). 
is necessary to make his preferences consistent with the Pigou-Dalton principle of transfers. Hence these results are distinct, but complementary.

Our main result can be stated as follows. By considering transfers having the structure of a discrete cone, and focusing on the utilitarian approach, if we are able to identify the class of utility functions which ensure the equivalence between statements of type (d) and (e) above (our Theorem 1), ${ }^{3}$ then we are sure that the equivalence between (a) and (b) in the HLP-type theorem is also true (our Theorem 2). It is important to emphasize that the first equivalence is easier to achieve. We also establish that the utilitarian approach in statement (b) can be extend to the largest class of social welfare functions consistent with the considered transfers. Thus our contribution is twofold. First, our result can be useful to complete theorems of the HLP-type, when the usual equivalences (a)-(c) or (b)-(c) are difficult to prove (see Section 6 for an illustration). Then, we put forward the particular role of the utilitarian approach in this context: An unanimous ranking of distributions within a utilitarian class is necessary, but also sufficient to reach unanimity among all the social welfare functions consistent with the considered transfers.

Literature. This paper builds on a large set of results in the theory of majorization (see Marshall et al., 2011) and a recent literature in the theory of decision under risk (Muller and Scarsini, 2012; Muller, 2013). The notion of 'quasi-ordering induced by a convex cone' has been introduced by Marshall et al. (1967) for a unidimensional real-valued variable. They establish, in this context, necessary and sufficient conditions to be placed on a real-valued function to be order-preserving (a result in line with the equivalence between (d) and (e) above). Marshall (1991) investigates, in the same framework, the equivalence between statements of type (a) and (b) above, by using duality arguments between a convex cone and its 'polar cone' (Proposition 3.4, Page 237). In decision under risk, Muller and Scarsini (2012) extend this result to multivariate probability distributions with real-valued dimensions, by defining a sequence of 'inframodular mass transfers' as a quasi-ordering induced by a convex cone. Muller (2013) generalizes this result to any transfers having the same structure. In this paper we propose a full discretization of this last result: outcomes are discrete and finite in each dimension, and a distribution simply counts the numbers of individuals in each outcome. Hence we define transfers as discrete cones, instead of convex cones. The arguments of the proof of our main result (Theorem 2) are almost the same but with an originality, the introduction of the notion of 'Hilbert basis'. We also extend the equivalence outside the utilitarian realm.

The rest of the paper is organized as follows. We introduce in Section 2 mathematical notions which are subsequently used for our formal definitions. We also recall some classic mathematical results, which underlie the proofs of our propositions and theorems. Hence the reader may start with the following sections, and then go back to this section when necessary. Section 3 is devoted to the presentation of our framework and main definitions, among of them an abstract notion of 'set of social welfare improving transfers'. Section 4 investigates social welfare functions consistent with the transfers

3 Actually, only '(e) implies (d)' has to be proved. With this proof in hand, the reverse implication can be easily obtained by using a proof by contrapositive. 
considered above, with a particular focus on the utilitarian class. Our main result is presented in Section 5, and illustrated in the next four sections. Section 6 considers 'Hammond transfers' in the case of a unidimensional ordinal variable. Section 7 deals with a bidimensional variable, consisting of a transferable (among the individuals) and cardinally measurable dimension, and a non-transferable ordinal dimension. Section 8 illustrates transfers outside our framework. Section 9 shows that our results can be of some interest for another economic field, the theory of decision under risk. Finally, Section 10 concludes.

\section{Mathematical preliminaries}

We denote by $\mathbb{Z}, \mathbb{Q}$ and $\mathbb{R}$, respectively, the sets of integers (including 0), rationals and real numbers. The symbols $\mathbb{Z}_{+}, \mathbb{Q}_{+}$and $\mathbb{R}_{+}$are the non-negative restrictions of these sets and $\mathbb{Z}_{++}, \mathbb{Q}_{++}$and $\mathbb{R}_{++}$, the positive restrictions. The cardinality of a set $\mathcal{A}$ is indicated by $|\mathcal{A}|$.

A convex cone is a nonempty set $\mathcal{C} \subset \mathbb{R}^{d}$ such that $c_{1}, c_{2} \in \mathcal{C}$ implies $\left(\lambda_{1} c_{1}+\right.$ $\left.\lambda_{2} c_{2}\right) \in \mathcal{C}$ for all $\lambda_{1}, \lambda_{2} \in \mathbb{R}_{+}$. We say that a convex cone is generated by a set $\mathcal{C}_{1} \subset \mathcal{C}$ if $\mathcal{C}=\operatorname{co}\left\{\lambda c \mid \lambda \in \mathbb{R}_{+}, c \in \mathcal{C}_{1}\right\}$, where co indicates the convex hull of the set. The convex cone $\mathcal{C}$ is finitely generated if it is generated by a finite set $\mathcal{C}_{1}=\left\{c_{1}, \ldots, c_{T}\right\}$ or, equivalently, if it can be written as $\mathcal{C}=\left\{\sum_{t=1}^{T} \lambda_{t} c_{t} \mid \lambda_{t} \in \mathbb{R}_{+}, c_{t} \in \mathcal{C}_{1}\right\}$. A rational cone is a convex cone $\mathcal{C} \subset \mathbb{R}^{d}$ finitely generated by a set $\left\{c_{1}, \ldots, c_{T}\right\}$ of elements in $\mathbb{Q}^{d}$. A discrete cone is the integer analogue of a convex cone. It is a nonempty set $\mathcal{C} \subset \mathbb{Z}^{d}$ such that $c_{1}, c_{2} \in \mathcal{C}$ implies $\left(\lambda_{1} c_{1}+\lambda_{2} c_{2}\right) \in \mathcal{C}$ for all $\lambda_{1}, \lambda_{2} \in \mathbb{Z}_{+}$. Finally a cone is said to be pointed if $c \in \mathcal{C}$ and $(-c) \in \mathcal{C}$ imply $c=0$.

For a convex cone $\mathcal{C} \subset \mathbb{R}^{d}$, a finite set $\left\{c_{1}, \ldots, c_{T}\right\}$ of elements in $\mathcal{C} \cap \mathbb{Z}^{d}$ is called a Hilbert basis if every $c \in \mathcal{C} \cap \mathbb{Z}^{d}$ can be expressed as $c=\sum_{t=1}^{T} \lambda_{t} c_{t}$ with $c_{t}$ in the basis and $\lambda_{t} \in \mathbb{Z}_{+}$. The set of all irreducible elements contained in the Hilbert basis of $\mathcal{C}$, that is, those elements that cannot be written as $c=c_{1}+c_{2}$ where $c_{1}, c_{2} \neq 0$ are elements of the basis, is called the minimal Hilbert basis, and denoted by $\mathscr{H}(\mathcal{C})$.

Lemma 1. If $\mathcal{C}$ is a rational cone, then it is generated by a minimal Hilbert basis $\mathscr{H}(\mathcal{C})$. Moreover if $\mathcal{C}$ is pointed, then $\mathscr{H}(\mathcal{C})$ is unique.

The existence part of the result has been first established by Gordan (1873) and Hilbert (1890). The unicity of $\mathscr{H}(\mathcal{C})$ as been shown by van der Corput (1931a,b) (see also Schrijver, 1986, Theorem 16.4, Page 233).

We then introduce some tools on binary relations. A binary relation $\succeq$ defined on a set $\mathcal{A}$ is a subset of $\mathcal{A} \times \mathcal{A}$. For all $c_{1}, c_{2} \in \mathcal{A}$, we write $c_{1} \succ c_{2}$ if and only if $c_{1} \succeq c_{2}$ and not $c_{2} \succeq c_{1}$. We let $c_{1} \sim c_{2}$ if and only if $c_{1} \succeq c_{2}$ and $c_{2} \succeq c_{1}$. The relation $\succeq$ is reflexive if $c \succeq c$, for all $c \in \mathcal{A}$. It is transitive if $c_{1} \succeq c_{2}$ and $c_{2} \succeq c_{3}$ imply $c_{1} \succeq c_{3}$, for all $c_{1}, c_{2}, c_{3} \in \mathcal{A}$. A reflexive and transitive relation is a quasi-ordering. It is antisymmetric if $c_{1} \sim c_{2}$ implies $c_{1}=c_{2}$, for all $c_{1}, c_{2} \in \mathcal{A}$. In this paper, a binary relation defined on $\mathcal{A}$ is said additive if $c_{1} \succeq c_{2}$ implies $c_{1}+c_{3} \succeq c_{2}+c_{3}$, for all $c_{1}, c_{2}, c_{3} \in \mathcal{A}$. It is said scale-invariant if $c_{1} \succeq c_{2}$ implies $\lambda c_{1} \succeq \lambda c_{2}$, for all $c_{1}, c_{2} \in \mathcal{A}$ 
and all $\lambda \in \mathbb{R}_{+}$. Finally, we say that a relation $\succeq$ on $\mathcal{A}$ is induced by the set $\mathcal{C}$ when $c_{1} \succeq c_{2}$ if and only if $\left(c_{1}-c_{2}\right) \in \mathcal{C}$. A relation $\succeq$ induced by a cone $\mathcal{C}$ is called a cone ordering. The following result is established in Marshall et al. (1967).

Lemma 2. A convex cone ordering, defined on $\mathbb{R}^{d}$, is an additive and scale-invariant quasi-ordering. Conversely, a quasi-ordering on $\mathbb{R}^{d}$ must be additive and scale-invariant, to be a convex cone ordering. Then, a convex cone is pointed if and only if the induced binary relation is antisymmetric.

We conclude this section with notions of duality between convex cones. This approach has been recently applied in economics by Muller and Scarsini (2012) and Muller (2013). Let $\mathcal{V}, \mathcal{W}$ be two vector spaces. A function $b: \mathcal{V} \times \mathcal{W} \rightarrow \mathbb{R}$ is called a bilinear mapping if it is linear in each argument separately, namely with $\lambda \in \mathbb{R}_{++}$:

$$
\begin{aligned}
& b(v+z, w)=b(v, w)+b(z, w) \quad \text { and } \quad b(\lambda v, w)=\lambda b(v, w) \\
& b(v, w+z)=b(v, w)+b(v, z) \quad \text { and } \quad b(v, \lambda w)=\lambda b(v, w) .
\end{aligned}
$$

The pair $(\mathcal{V}, \mathcal{W})$ of vector spaces is said to be in duality if there exists a bilinear mapping $b: \mathcal{V} \times \mathcal{W} \rightarrow \mathbb{R}$. We denote by $(\mathcal{V}, \mathcal{W} ; b)$ such a dual pair. The duality is said to be strict, if for each $0 \neq v \in \mathcal{V}$ there is a $w \in \mathcal{W}$ with $b(v, w) \neq 0$ and, for each $0 \neq w \in \mathcal{W}$, there is a $v \in \mathcal{V}$ with $b(v, w) \neq 0$. The polar cone of $\mathcal{V}_{1} \subset \mathcal{V}$ under the duality $(\mathcal{V}, \mathcal{W} ; b)$ is defined by:

$$
\mathcal{V}_{1}^{\circ}=\left\{w \in \mathcal{W} \mid b(v, w) \leq 0, \forall v \in \mathcal{V}_{1}\right\}
$$

Notice that $\mathcal{V}_{1}^{\circ}$ is always a convex cone even if $\mathcal{V}_{1}$ is neither convex, nor a cone. The bipolar cone of the $\mathcal{V}_{1}$ is denoted $\mathcal{V}_{1}^{\circ \circ}$ and defined by $\mathcal{V}_{1}^{\circ \circ}=\left(\mathcal{V}_{1}^{\circ}\right)^{\circ}$. The bipolar theorem can be stated as follows (see Rockafellar, 1970; Muller, 2013):

Lemma 3. Assume that $\mathcal{V}, \mathcal{W}$ are two vector spaces in strict duality and let $\mathcal{V}_{1} \subset \mathcal{V}$ Then $\mathcal{V}_{1}^{\circ \circ}$ is the smallest closed set containing the convex cone generated by $\mathcal{V}_{1}$.

As a particular case of the previous theorem, notice that $\mathcal{V}_{1} \subset \mathcal{V}$ is a nonempty closed convex cone if and only if $\mathcal{V}_{1}^{\circ \circ}=\mathcal{V}_{1}$.

\section{Outcomes, distributions and social welfare improving transfers}

The set of outcomes is assumed to be a partially ordered, finite and fixed set $\mathcal{S} \subset \mathbb{Z}_{+}^{d}$. Hence we assume that each individual in the society is characterized by an outcome in $d$-dimensions (income, health, ...), such that outcomes in one dimension are ordered (according to the usual ordering on $\mathbb{Z}$ ), but only partially ordered in a multidimensional setting. The second and last restriction is that each dimension is assumed to be finitely divisible. A distribution is described by a list $n=\left(n_{s}\right)_{s \in \mathcal{S}}$, where $n_{s} \in \mathbb{Z}_{+}$indicates the number of individuals having ( $d$-dimensional) outcome $s \in \mathcal{S}$. We denote by $\mathcal{N}=\left\{n \in \mathbb{Z}_{+}^{|\mathcal{S}|} \mid \sum_{s \in \mathcal{S}} n_{s}=N\right\}$ the set of distributions for populations of fixed size 
$N .{ }^{4}$ We provide in Figure 2 a simple example where outcomes are bidimensional, hence dots $s=(i, j) \in \mathbb{Z}_{+}^{2}$, and where a distribution is simply a list of $n_{(i, j)}$.

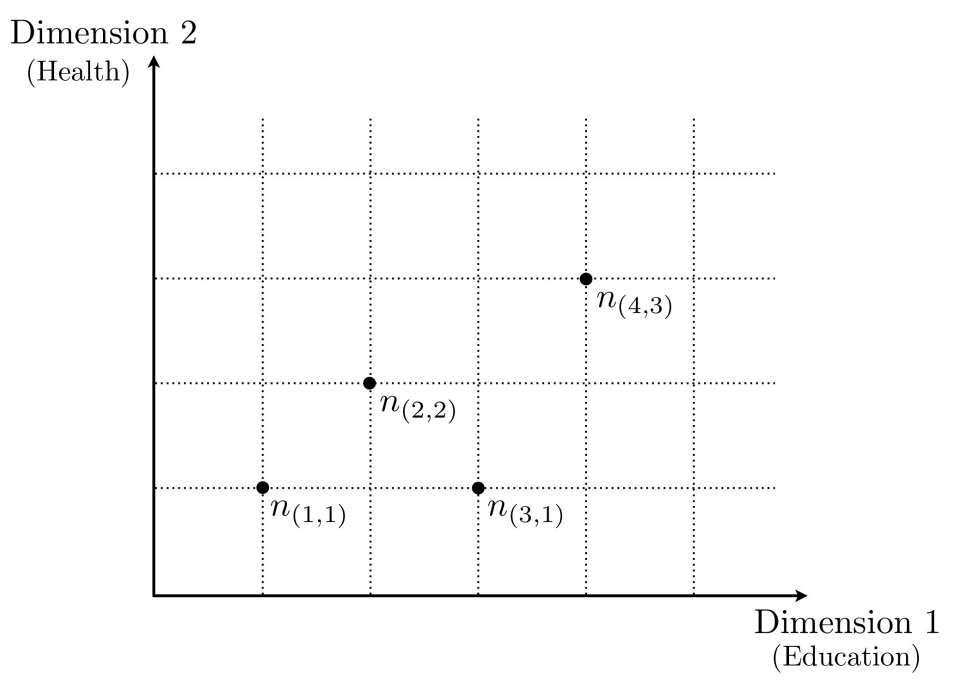

Figure 1: Example of a bidimensional variable

We emphasize that this framework is sufficiently large to encompass most of the usual frameworks investigated in the literature of inequality, poverty and social welfare measurement. For instance each dimension can be, independently, defined on a cardinal or ordinal scale. Income is an example of a possible cardinal outcome dimension. In that case, in order to satisfy the finite divisibility assumption, one has to consider euro cent as the smallest income unit (which is, in practice, always assumed). Our approach is illustrated with specific examples in Sections 6 and 7.

We now provide a general definition of a set of transfers (which can be, at this stage, welfare-improving or not). This definition is provided at an abstract level, in the sense that it only specifies fundamental properties such a set could satisfy. As illustrated in Sections 6 and 7, most of the sets of transfers usually considered in the literature (increments, Pigou-Dalton progressive transfers, ... ) are well-described as particular case of our general definition. The main idea is as follows. Consider two distributions $n, n^{\prime} \in \mathcal{N}$, and assumed that $n$ is obtained from $n^{\prime}$ by means of a finite sequence of transfers. A transfer is just a movement of one individual from one outcome to another. Such a sequence of transfers can be written by means of a vector $m=n-n^{\prime}$, which lists the differences between the number of individuals before and after the transfers, in each outcome $s \in \mathcal{S}$.

We first introduce some technicalities. For any vector $m \in \mathbb{R}^{|\mathcal{S}|}$, we denote $m^{+}=$ $\left(m_{s}^{+}\right)_{s \in \mathcal{S}}$ where $m_{s}^{+}=\max \left(m_{s}, 0\right)$ and $m^{-}=\left(m_{s}^{-}\right)_{s \in \mathcal{S}}$ where $m_{s}^{-}=\max \left(-m_{s}, 0\right)$, such that $m=m^{+}-m^{-}$. We define by $\mathcal{M}$ the set of all vectors $m \in \mathbb{R}^{|\mathcal{S}|}$ such that

${ }^{4}$ By applying the Dalton's principle of populations which assumes that an identical replication of the population is distributionally equivalent to the initial one we only consider, without loss of generality, distributions in $\mathcal{N}$. 
$\sum_{s \in \mathcal{S}} m_{s}^{+}=\sum_{s \in \mathcal{S}} m_{s}^{-}<\infty$. The sets of transfers we consider in this paper are subsets of $\mathcal{M}$.

Definition 1 (Set of transfers). The set $\mathcal{T}$ of transfers is the set of all $m \in \mathbb{Z}^{|\mathcal{S}|}$ which satisfies the following two properties:

(i) There exist $n, n^{\prime} \in \mathcal{N}$ such that $m$ can be written as $m=n-n^{\prime}$,

(ii) $m \in \mathcal{T}$ and $(-m) \in \mathcal{T}$ imply $m=0$.

This definition is illustrated by the standard notion of increment. In the bidimensional case, we say that distribution $n$ is obtained from distribution $n^{\prime}$ by means of a (weak) increment, if and only if $n_{s}=n_{s}^{\prime}$ for all $s \in \mathcal{S} \subset \mathbb{Z}_{+}^{2}$, or if there exist $(i, j),(k, l) \in \mathcal{S}$ such that: ${ }^{5}$

$$
\begin{gathered}
n_{s}=n_{s}^{\prime}, \text { for all } s \neq(i, j),(k, l), \\
n_{(i, j)}=n_{(i, j)}^{\prime}-1, n_{(k, l)}=n_{(k, l)}^{\prime}+1, \text { and }(i, j)<(k, l) .
\end{gathered}
$$

That corresponds to a population move towards the northeast in the graph of Figure 2 . We can define the set of increments, denoted $\mathcal{T}_{I}$, by the set all vectors $m \in \mathbb{Z}^{|\mathcal{S}|}$ such that, either $m_{s}=0$ for all $s \in \mathcal{S} \subset \mathbb{Z}_{+}^{2}$, or there exist $(i, j),(k, l) \in \mathcal{S}$ such that:

$$
\begin{gathered}
m_{s}=0, \text { for all } s \neq(i, j),(k, l), \\
m_{(i, j)}=-1, m_{(k, l)}=1 \text {, and }(i, j)<(k, l) .
\end{gathered}
$$

If an increment is assumed to be welfare-improving, the reverse transfer, which corresponds to $(-m)$ in the previous definition, is welfare-decreasing and called a decrement. Clearly, $\mathcal{T}_{I} \subset \mathcal{T}$.

The last distinction between increments and decrements illustrates, and justifies property (ii) in Definition 1. Indeed, a welfare-improving transfer is in some sense directional: If $m \in \mathcal{T}$ is considered as a welfare improvement, then it cannot be also true for $(-m)$ unless $m=0$. Notice that transfers are here considered in a weak sense, as statu quo is allowed: This is a consequence of property (i) and the fact that $m=0 \in \mathcal{T}$ (we can write $0=n-n$ ). Another implication of property (i) is that $\mathcal{T}$ is a discrete and finite set. Moreover, for any $m \in \mathcal{T}$ one also has $\sum_{s \in \mathcal{S}} m_{s}=0$, a consequence of the fixed population size characterizing distributions $n, n^{\prime} \in \mathcal{N}$. Because $\sum_{s \in \mathcal{S}} m_{s}=\sum_{s \in \mathcal{S}} m_{s}^{+}-\sum_{s \in \mathcal{S}} m_{s}^{-}$, it follows that $\sum_{s \in \mathcal{S}} m_{s}^{+}=\sum_{s \in \mathcal{S}} m_{s}^{-}(\leq N)$. This remark confirms that $\mathcal{T} \subset \mathcal{M}$.

Even if Definition 1 is sufficiency flexible to encompass most of the welfare-improving transfers considered in the literature, it imposes some restrictions, among which the following one.

Remark 1 (Independence). Let $n, n^{\prime}, m, m^{\prime} \in \mathcal{N}$, such that $m=n+\epsilon$ and $m^{\prime}=n^{\prime}+\epsilon$. If $n$ is obtained from $n^{\prime}$ by means of a transfer in $\mathcal{T}$, then $m$ is also obtained from $m^{\prime}$ by means of a transfer in $\mathcal{T}$.

5 Vector inequalities are denoted $\leq,<$ and $\ll$. 


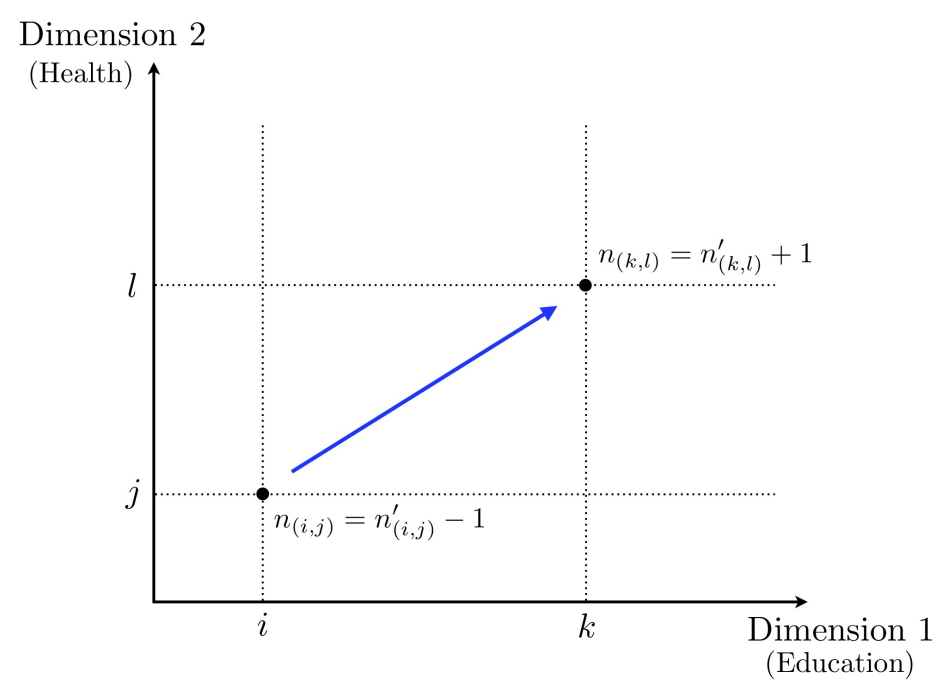

Figure 2: An increment

This observation trivially follows from the fact that, if $n, n^{\prime} \in \mathcal{N}$ and $\left(n-n^{\prime}\right) \in \mathcal{T}$, then $\left(m-m^{\prime}\right) \in \mathcal{T}$ for all $m, m^{\prime} \in \mathcal{N}$ such that $\left(n-n^{\prime}\right)=\left(m-m^{\prime}\right)$. Remark 1 has important implications: It states that admissible transfers in $\mathcal{T}$ are, necessarily, not impacted by the common parts of the distributions under comparison. This independence requirement is not satisfied by some sets of transfers considered in the literature, as illustrated in Section 8.

If the set of welfare-improving transfers we are interested in can be adequately written as in Definition 1, one can infer from $\mathcal{T}$ a dominance relation which can help a social evaluator to rank distributions in $\mathcal{N}$. Indeed, if one distribution is obtained from another by a sequence of welfare-improving transfers, then the social evaluator can unambiguously consider that the first one is socially better. If $\mathcal{T}$ defines the admissible transfers, then the discrete cone generated by $\mathcal{T}$ is the natural way to define a sequence of such transfers. ${ }^{6}$

Definition 2 (Sequence of transfers in $\mathcal{T}$ ). For all $m, m^{\prime} \in \mathbb{Z}^{|\mathcal{S}|}$, we write $m \succeq_{\mathcal{T}} m^{\prime}$ if and only if $\succeq_{\mathcal{T}}$ is induced by the discrete cone generated by $\mathcal{T}$. Formally, $m \succeq_{\mathcal{T}} m^{\prime}$ if and only if $\left(m-m^{\prime}\right) \in \mathcal{D}(\mathcal{T})$, where $\mathcal{D}(\mathcal{T})=\left\{\sum_{t=1}^{|\mathcal{T}|} \lambda_{t} m_{\cdot_{t}} \mid \lambda_{t} \in \mathbb{Z}_{+}, m_{\cdot t} \in \mathcal{T}\right\}$ with $m_{\cdot t}=\left(m_{s t}\right)_{s \in \mathcal{S}}$.

We emphasize that the relation $\succeq_{\mathcal{T}}$ is defined on $\mathbb{Z}^{|\mathcal{S}|}$ instead of $\mathcal{N}$ even if it is only used, in this paper, to compare distributions in $\mathcal{N}$. That is a consequence of basic properties of a discrete cone. For instance we have, by definition, $m \succeq_{\mathcal{T}} m^{\prime}$ if and only if $\left(m-m^{\prime}\right) \succeq_{\mathcal{T}} 0$. The following remark states that, at least, the equal size condition imposed to distributions in $\mathcal{N}$, is also required to distributions $m, m^{\prime} \in \mathbb{Z}^{|\mathcal{S}|}$ when compared by $\succeq_{\mathcal{T}}$.

6 The use of the notion of discrete cone justifies the implicit requirement that $0 \in \mathcal{T}$ in Definition 1. 
Remark 2 (Same population size). A necessary condition for $m \succeq_{\mathcal{T}} m^{\prime}$ is that $\sum_{s \in \mathcal{S}} m_{s}=\sum_{s \in \mathcal{S}} m_{s}^{\prime}<\infty$.

Proof. From (i) in Definition 1, we know that $m=\left(m_{s}\right)_{s \in \mathcal{S}} \in \mathcal{T}$ implies $\sum_{s \in \mathcal{S}} m_{s}=0$. This last equality is thus also true for all $m \in \mathcal{D}(\mathcal{T})$. Because $m \succeq_{\mathcal{T}} m^{\prime}$ if and only if $\left(m-m^{\prime}\right) \in \mathcal{D}(\mathcal{T}), m \succeq_{\mathcal{T}} m^{\prime}$ implies $\sum_{s \in \mathcal{S}}\left(m_{s}-m_{s}^{\prime}\right)=0$. Finally, we have previously established that $\sum_{s \in \mathcal{S}} m_{s}^{+}=\sum_{s \in \mathcal{S}} m_{s}^{-} \leq N$, hence $\sum_{s \in \mathcal{S}} m_{s}=\sum_{s \in \mathcal{S}} m_{s}^{\prime}<\infty$.

We now present the main properties satisfied by $\succeq_{\mathcal{T}}$. This Proposition is a mere transposition of Lemma 2 to our discrete cones, noting that Equation (8) is a weak scale-invariance property, as it only applies to scale parameter in $\mathbb{Z}_{+}$(instead of $\mathbb{R}_{+}$).

Proposition 1. The discrete cone ordering $\succeq_{\mathcal{T}}$ is an additive quasi-ordering, and satisfies the following property:

$$
\forall m, m^{\prime} \in \mathbb{Z}^{|\mathcal{S}|}: \quad m \succeq_{\mathcal{T}} m^{\prime} \Longrightarrow \lambda m \succeq_{\mathcal{T}} \lambda m^{\prime}, \quad \forall \lambda \in \mathbb{Z}_{+} .
$$

As it is also antisymmetric, the discrete cone $\mathcal{D}(\mathcal{T})$ is pointed.

Proof. For any $m \in \mathbb{Z}^{|\mathcal{S}|}$, we have $(m-m)=0 \in \mathcal{D}(\mathcal{T})$. Hence $m \succeq_{\mathcal{T}} m$ so that $\succeq_{\mathcal{T}}$ is reflexive. Now suppose that $m \succeq_{\mathcal{T}} m^{\prime}$ and $m^{\prime} \succeq_{\mathcal{T}} m^{\prime \prime}$. It follows that $\left(m-m^{\prime}\right) \in \mathcal{D}(\mathcal{T})$ and $\left(m^{\prime}-m^{\prime \prime}\right) \in \mathcal{D}(\mathcal{T})$. Moreover $\left(m-m^{\prime \prime}\right)=\left(m-m^{\prime}\right)+\left(m^{\prime}-m^{\prime \prime}\right) \in \mathcal{D}(\mathcal{T})$, or equivalently $m \succeq_{\mathcal{T}} m^{\prime \prime}$. Hence $\succeq_{\mathcal{T}}$ si transitive. As it is reflexive and transitive, it is a quasi-ordering. Additivity is immediately obtained by noting that $\left(m-m^{\prime}\right) \in \mathcal{D}(\mathcal{T})$ if and only if $\left(\left(m+m^{\prime \prime}\right)-\left(m^{\prime}+m^{\prime \prime}\right)\right) \in \mathcal{D}(\mathcal{T})$. The implication in Equation (8) also follows from the definition of a discrete cone, so that $\left(m-m^{\prime}\right) \in \mathcal{D}(\mathcal{T})$ implies $\lambda\left(m-m^{\prime}\right) \in \mathcal{D}(\mathcal{T})$, as soon as $\lambda \in \mathbb{Z}_{+}$. In order to prove antisymmetry, suppose that $m \succeq_{\mathcal{T}} m^{\prime}$ and $m^{\prime} \succeq_{\mathcal{T}} m$, so that $\left(m-m^{\prime}\right) \in \mathcal{D}(\mathcal{T})$ and $\left(m^{\prime}-m\right) \in \mathcal{D}(\mathcal{T})$. Hence $\left(m-m^{\prime}\right)=\sum_{t=1}^{|\mathcal{T}|} \lambda_{t} m_{\cdot t}$ for some $\lambda_{t} \in \mathbb{Z}_{+}$and $m_{\cdot t} \in \mathcal{T}$. Moreover $\left(m^{\prime}-m\right)=-\left(m-m^{\prime}\right)=\sum_{t=1}^{|\mathcal{T}|} \lambda_{t}\left(-m_{. t}\right)$ and because $\left(m^{\prime}-m\right) \in \mathcal{D}(\mathcal{T})$, we have $\left(-m_{\cdot t}\right) \in \mathcal{T}$. Since $m_{\cdot t} \in \mathcal{T}$ and $\left(-m_{\cdot t}\right) \in \mathcal{T}$, it follows that $m_{\cdot t}=0$ for all $t=1, \ldots, T$, from Definition 1 . Hence $m=m^{\prime}$, so that $\succeq_{\mathcal{T}}$ is antisymmetric. As $\mathcal{T} \subseteq \mathcal{D}(\mathcal{T})$, the previous argument equivalently states that $\mathcal{D}(\mathcal{T})$ is pointed.

Proposition 1 has some implications when distributions $n, n^{\prime} \in \mathcal{N}$ are compared. Following Equation (8), if $n \succeq_{\mathcal{T}} n^{\prime}$, then an identical replication of the population has no impact on the ranking of the distributions $n$ and $n^{\prime}$. This property is thus related to the Dalton's principle of populations, but in a weaker sense since it does not required that $n \sim_{\mathcal{T}} \lambda n$. Indeed, as $\succeq_{\mathcal{T}}$ is antisymmetric, we have $n \sim_{\mathcal{T}} n^{\prime}$ if and only if $n=n^{\prime}$. We conclude that Equation (8) is a suitable property, because standard in normative economics. Additivity is somewhat stronger, and related to the independence condition as described in Remark 1 (see Section 8 for a discussion).

We now formally define the notion of minimality of a set of transfers. Welfareimproving transfers are usually defined in such way that they involve a small number of individuals, in order to be transparent on their ethical meaning. But the transparency of a transfer does not guarantee that the transformation underlying the transfer is 
minimal from a mathematical point of view. This distinction is important when we want to test whether one distribution is obtained (or not) from another by a sequence of admissible transfers.

Definition 3 (Minimal transfers). We say that the set of transfers $\mathcal{T}$ is minimal if and only if it is a set of irreducible elements, that is the set of transfers which share the properties of $\mathcal{T}$ (see Definition 1), but which cannot be written as a positive linear combination of other transfers in $\mathcal{T}$.

As an illustration the set $\mathcal{T}_{I}$ of increments, introduced after Definition 1 , is not defined as a minimal set. Indeed, one immediately observes that increments are minimal if and only if the pair $(i, j),(k, l) \in \mathbb{Z}_{+}^{2}$ is defined in such way that $(k, l)=(i+1, j)$ or $(k, l)=(i, j+1)$. Any transfer in $\mathcal{T}_{I}$ can be written as a positive linear combination of such minimal transfers.

\section{Social welfare functions}

The previous section provides an abstract model of welfare-improving transfers which encompass most of the transfers considered in the literature (such as, for instance, increments and Pigou-Dalton progressive transfers). That leads to a quasi-ordering which can be used to rank distributions of individuals, according to the outcomes they have. In this section we investigate another approach to compare distributions, building on the preferences of a social planner, or a group of social planners. Such preferences are characterized by a set of ethical considerations, which are not restricted to the mere notion a welfare-improving transfers. The ambition is here to introduce a quasi-ordering which reflects the unanimity of rankings among a large class of social planners, sharing some ethical views.

We first assume that the preferences of a social planner can be represented by a social welfare function $W: \mathbb{Z}^{|\mathcal{S}|} \rightarrow \mathbb{R}$. Again $W$ is defined on $\mathbb{Z}^{|\mathcal{S}|}$, but it will only be used to compare distributions in $\mathcal{N}$. We denote by $\mathcal{W}$ the set of all these social welfare functions. We now focus on the social welfare functions consistent with $\succeq_{\mathcal{T}}$, or equivalently on all the social planners who agree that transfers in $\mathcal{T}$ are unambiguously welfare-improving.

Definition 4 (Consistency with $\succeq_{\mathcal{T}}$ ). We say that $W \in \mathcal{W}$ is consistent with $\succeq_{\mathcal{T}}$ if and only if, for all $m, m^{\prime} \in \mathbb{Z}^{|\mathcal{S}|}, m \succeq_{\mathcal{T}} m^{\prime} \Longrightarrow W(m) \geq W\left(m^{\prime}\right)$. The set of all functions $W \in \mathcal{W}$ consistent with $\succeq_{\mathcal{T}}$ is written $\mathcal{W}_{\mathcal{T}}{ }^{7}{ }^{7}$

We emphasize that $\mathcal{W}_{\mathcal{T}}$ identifies the set of all social planners sharing the ethical views captured by the transfers in $\mathcal{T}$, but their preferences are not necessarily equivalent elsewhere.

Considering two distributions $m, m^{\prime} \in \mathbb{Z}^{|\mathcal{S}|}$, one immediately deduces from Definition 4 that $W \in \mathcal{W}_{\mathcal{T}}$ and $m \succeq_{\mathcal{T}} m^{\prime}$ is sufficient to have $W(m) \geq W\left(m^{\prime}\right)$. We establish

7 Notice that a function consistent with a quasi-ordering is sometimes called order-preserving (see Marshall et al., 1967). 
in the following result that $m$ weakly better that $m^{\prime}$ according to all the social welfare functions in $\mathcal{W}_{\mathcal{T}}$ is, actually, also sufficient to have $m \succeq_{\mathcal{T}} m^{\prime}$.

Proposition 2. For all $m, m^{\prime} \in \mathbb{Z}^{|\mathcal{S}|}$ such that $\sum_{s \in \mathcal{S}} m_{s}=\sum_{s \in \mathcal{S}} m_{s}^{\prime}<\infty$, the following two statements are equivalent: ${ }^{8}$

(a) $m \succeq_{\mathcal{T}} m^{\prime}$,

(b) $W(m) \geq W\left(m^{\prime}\right), \forall W \in \mathcal{W}_{\mathcal{T}}$,

Proof. The implication $(a) \Rightarrow(b)$ follows directly from Definition 4 . The argument to establish $(b) \Rightarrow(a)$ exactly follows Marshall (1991), Proposition 2.8, Page 234. First, recall that $m \succeq_{\mathcal{T}} m^{\prime}$ if and only if $\left(m-m^{\prime}\right) \in \mathcal{D}(\mathcal{T})$, where $\mathcal{D}(\mathcal{T})$ is the discrete cone generated by $\mathcal{T}$.

For all $z \in \mathbb{Z}^{|\mathcal{S}|}$, choose $W_{m}(z)=0$ if $(m-z) \in \mathcal{D}(\mathcal{T})$, and $W_{m}(z)=1$ otherwise. We first establish that $W_{m} \in \mathcal{W}_{\mathcal{T}}$. Consider $u, v \in \mathbb{Z}^{|\mathcal{S}|}$ and assume that $u \succeq_{\mathcal{T}} v$. If $W_{m}(u)=1$, then $W_{m}(u) \geq W_{m}(v)$ because, by definition, $W_{m}(v) \in\{0,1\}$. Now suppose that $W_{m}(u)=0$, or equivalently $(m-u) \in \mathcal{D}(\mathcal{T})$. Because $u \succeq_{\mathcal{T}} v$, we also have $(u-v) \in \mathcal{D}(\mathcal{T})$. As $\mathcal{D}(\mathcal{T})$ is a discrete cone, it follows that $(m-u)+(u-v)=$ $(m-v) \in \mathcal{D}(\mathcal{T})$. Hence $W_{m}(v)=0$ so that, again, $W_{m}(u) \geq W_{m}(v)$. We conclude that $u \succeq_{\mathcal{T}} v$ implies $W_{m}(u) \geq W_{m}(v)$. Hence, $W_{m} \in \mathcal{W}_{\mathcal{T}}$.

Now assume that statement (b) is true. It follows that $W_{m}(m) \geq W_{m}\left(m^{\prime}\right)$. By definition $(m-m)=0 \in \mathcal{D}(\mathcal{T})$, hence $W_{m}(m)=0$. That implies that, also, $W_{m}\left(m^{\prime}\right)=0$ or equivalently $\left(m-m^{\prime}\right) \in \mathcal{D}(\mathcal{T})$. Thus $m \succeq_{\mathcal{T}} m^{\prime}$.

The previous proposition allows to go a step further to compare distributions $m$ and $m^{\prime}$ according to $\succeq_{\mathcal{T}}$, as it establishes the equivalence with an, a priori, distinct quasiordering based on the unanimity of rankings among a group of social planners. Nevertheless, this result is not really informative. As $\mathcal{W}_{\mathcal{T}}$ encompasses a large class of social welfare functions, the required unanimity can not be checked. Indeed, the set $\mathcal{W}_{\mathcal{T}}$ is (uncountably) infinite because, by definition, any strictly increasing transformation of a function $W \in \mathcal{W}_{\mathcal{T}}$ is also in $\mathcal{W}_{\mathcal{T}}$.

One first observes that $\mathcal{W}_{\mathcal{T}}$ has the structure of a convex cone. Because $\mathcal{W}_{\mathcal{T}}$ is defined as the largest set of functions consistent with $\succeq_{\mathcal{T}}$, if $W, W^{\prime} \in \mathcal{W}_{\mathcal{T}}$, then $\left(\lambda W+\lambda^{\prime} W^{\prime}\right) \in \mathcal{W}_{\mathcal{T}}$ for all $\lambda, \lambda^{\prime} \in \mathbb{R}_{+}$. It follows that one objective can be to identify the minimal set of social welfare functions (namely, the basis) which characterizes $\mathcal{W}_{\mathcal{T}}$, if such a set exists. Then, one notices that Proposition 2 establishes an equivalence between two quasi-orderings, but of different nature. Whereas the quasi-ordering in statement (b) is generated by a convex cone, the quasi-ordering in statement (a) is generated by a discrete cone, which moreover satisfies an independence property (Remark 1). ${ }^{9}$

8 We recall that $\sum_{s \in \mathcal{S}} m_{s}=\sum_{s \in \mathcal{S}} m_{s}^{\prime}<\infty$ is a necessary condition for $m \succeq_{\mathcal{T}} m^{\prime}$ (see Remark 2), which justifies the initial statement of the proposition.

9 The fact that two cone orderings of different nature appear to be equivalent does not come as a surprise. By using the terminology of Marshall (1991), the cone ordering induced by $\mathcal{W}_{\mathcal{T}}$ is called the completion of $\succeq_{\mathcal{T}}$. 
In what follows we focus on the utilitarian social welfare functions, the prevailing approach in the literature to compare distributions. Because the sets of welfareimproving transfers we consider are discrete cones this subclass of $\mathcal{W}_{\mathcal{T}}$ plays, actually, a particular role. Indeed, we establish that it is possible to identify a class of utilitarian social welfare functions such that a unanimity of ranking among this class is necessary but also sufficient to have dominance according to $\succeq_{\mathcal{T}}$. Intuitively, the independence property satisfied by the discrete cones and the fact that the utilitarian approach involved additive separable functions can, at least partially, explain this relationship.

We denote by $u=\left(u_{s}\right)_{s \in \mathcal{S}} \in \mathbb{R}^{|\mathcal{S}|}$ a vector which assigns utility $u_{s} \in \mathbb{R}$ to any outcome $s \in \mathcal{S}$, and we denote by $\mathcal{U}$ the set of all such vectors defined up to an increasing affine transformation. A utilitarian social welfare function $W_{u}: \mathbb{Z}^{|\mathcal{S}|} \rightarrow \mathbb{R}$ is defined by $W_{u}(m)=\sum_{s \in \mathcal{S}} m_{s} u_{s}$, for all $m \in \mathbb{Z}^{|\mathcal{S}|}$ and all $u \in \mathcal{U}$. We now adapt the notion of consistency with $\succeq_{\mathcal{T}}$ (Definition 4) to this class of functions. We first define:

$$
\mathcal{U}_{\mathcal{T}}=\left\{u \in \mathcal{U} \mid \sum_{s \in \mathcal{S}} m_{s} u_{s} \geq 0, \forall m \in \mathcal{T}\right\}
$$

One immediately observes that the consistency of a utilitarian social welfare function with $\succeq_{\mathcal{T}}$ is obtained if and only if $u \in \mathcal{U}_{\mathcal{T}}$, as stated in the following theorem. The degree of generality is still high, and the proof quite obvious. This proof is nevertheless provided as it can help at establishing other results, especially in the case of specific classes of transfers in $\mathcal{T}$. It is presented as a theorem because the core of this paper is to establish that, for specific classes of transfers, being able to identify the subclass of $\mathcal{U}$ such that Theorem 1 is true, is sufficient to prove a result like Theorem 2.

Theorem 1. The following two statements are equivalent:

(a) $\forall m, m^{\prime} \in \mathbb{Z}^{|\mathcal{S}|}: \quad m \succeq_{\mathcal{T}} m^{\prime} \Longrightarrow W_{u}(m) \geq W_{u}\left(m^{\prime}\right)$,

(b) $u \in \mathcal{U}_{\mathcal{T}}$.

Proof. We first prove that $(b) \Rightarrow(a)$. Assume that $u \in \mathcal{U}_{\mathcal{T}}$ and $m \succeq_{\mathcal{T}} m^{\prime}$, or equivalently $\left(m-m^{\prime}\right) \in \mathcal{D}(\mathcal{T})$. Hence there exist $T \leq|\mathcal{T}|, \lambda_{t} \in \mathbb{Z}_{++}$and $m_{. t} \in \mathcal{T}$ such that $\left(m-m^{\prime}\right)=\sum_{t=1}^{T} \lambda_{t} m_{\cdot t}$. It follows that $W_{u}(m)-W_{u}\left(m^{\prime}\right)=\sum_{s \in \mathcal{S}}\left(m_{s}-m_{s}^{\prime}\right) u_{s}=$ $\sum_{t=1}^{T} \lambda_{t}\left(\sum_{s \in \mathcal{S}} m_{s t} u_{s}\right)$. Because $u \in \mathcal{U}_{\mathcal{T}}$ and $m_{\cdot t} \in \mathcal{T}$ one has $\sum_{s \in \mathcal{S}} m_{s t} u_{s} \geq 0$, for all $t=1,2, \ldots, T$. Thus $W_{u}(m) \geq W_{u}\left(m^{\prime}\right)$.

To prove the reverse implication, we prove that $\neg(b) \Rightarrow \neg(a)$. Assume that $u \notin \mathcal{U}_{\mathcal{T}}$, so that there exists a $m^{\prime \prime} \in \mathcal{T}$ such that $\sum_{s \in \mathcal{S}} m_{s}^{\prime \prime} u_{s}<0$. Choose $m, m^{\prime} \in \mathbb{Z}^{|\mathcal{S}|}$ such that $\left(m-m^{\prime}\right)=m^{\prime \prime}$. Clearly $m \succeq_{\mathcal{T}} m^{\prime}$, but $W_{u}(m)-W_{u}\left(m^{\prime}\right)=\sum_{s \in \mathcal{S}}\left(m_{s}-m_{s}^{\prime}\right) u_{s}=$ $\sum_{s \in \mathcal{S}} m_{s}^{\prime \prime} u_{s}<0$. Hence there exist $m, m^{\prime} \in \mathbb{Z}^{|\mathcal{S}|}$ such that $m \succeq_{\mathcal{T}} m^{\prime}$ and $W_{u}(m)<$ $W_{u}\left(m^{\prime}\right)$, so that $(a)$ is false.

We conclude this section by first noting that constant utilities, such that $u_{s}=c$ for all $s \in \mathcal{S}$ and some $c \in \mathbb{R}$, belong to $\mathcal{U}_{\mathcal{T}}$. We then observes that $\mathcal{U}_{\mathcal{T}}$ is also a convex cone, as expected. Indeed, if $u, u^{\prime} \in \mathcal{U}_{\mathcal{T}}$, then $\left(\lambda u+\lambda^{\prime} u^{\prime}\right) \in \mathcal{U}_{\mathcal{T}}$ for all $\lambda, \lambda^{\prime} \in \mathbb{R}_{+}$. 


\section{Main result}

Whereas it was pretty obvious to establish that the unanimity of ranking between $m$ on $m^{\prime}$ among all $W \in \mathcal{W}_{\mathcal{T}}$ is a necessary and sufficient condition to have $m \succeq_{\mathcal{T}} m^{\prime}$ (Proposition 2), such a result is not so clear when the unanimity among all utilitarian social welfare functions is only achieved. Indeed, the class of utilitarian social welfare functions is a strict subclass of $\mathcal{W}_{\mathcal{T}}$ - the set of all social welfare functions consistent with $\succeq_{\mathcal{T}}$ - and it is not obvious that such a unanimity, in some sense relatively small, is sufficient to have $m \succeq_{\mathcal{T}} m^{\prime}$. This is what we prove here, as the main result of the paper. ${ }^{10}$ We first need a preliminary result. We recall that the set of distributions $\mathcal{N}$ is such that $\mathcal{N} \in \mathcal{M}$, where $\mathcal{M}$ is defined as the set of all vectors $m \in \mathbb{R}^{|\mathcal{S}|}$ with finite variation (see Section 3).

Lemma 4. $(\mathcal{M}, \mathcal{U} ; b)$ is a strict dual pair, where $b(m, u)=-\sum_{s \in \mathcal{S}} m_{s} u_{s}$.

Proof. $\mathcal{M}$ and $\mathcal{U}$ are two vector spaces, and $b: \mathcal{M} \times \mathcal{U} \rightarrow \mathbb{R}$ is a bilinear mapping. Hence $(\mathcal{M}, \mathcal{U} ; b)$ is a dual pair. It remains to prove that it is also strict. Because $\left|m^{+}\right|=\left|m^{-}\right|$for all $m \in \mathcal{M}$, one observes that, if $u=(\alpha, \ldots, \alpha) \in \mathcal{U}$, then $b(m, u)=0$ for all $m \in \mathcal{M}$. Nevertheless, as $u$ is definition up to an increasing affine transformation, $\mathcal{U}$ is a quotient space, that is the set of all equivalent classes $[v]=\left\{u \in \mathbb{R}^{|\mathcal{S}|} \mid u \sim v\right\}$, with the equivalence relation $u \sim v \Leftrightarrow u=a v+b$ $(a>0, b \in \mathbb{R})$. Hence, any constant utility $u=(\alpha, \ldots, \alpha)$ is in the equivalent class of $u=(0, \ldots, 0)$. One deduces that for each $0 \neq u \in \mathcal{U}$ there is a $m \in \mathcal{M}$ with $b(m, u) \neq 0$ and for each $0 \neq m \in \mathcal{M}$ there is a $u \in \mathcal{U}$ with $b(m, u) \neq 0$. One concludes that $(\mathcal{M}, \mathcal{U} ; b)$ is a strict dual pair. See Marshall et al. (2011), Page 99 or Muller (2013), Page 50, for related discussions.

We are now ready to present our main result. Equivalence between statements (a) and (b) in Theorem 2 below is a full discretization of Theorem 2.4.1. provided by Muller (2013), Page 51. Indeed our distribution are discrete in the sense that we only count individuals (value in $\mathbb{Z}$ ) in each possible discrete and finite outcome, whereas Muller (2013) considers probability measures (value in $\mathbb{R}$ ). It follows that the sequence of transfers we consider is defined as a discrete cone, not a convex cone. Consequently, we do not have to refer to a weak closure argument for the cone we consider, as done by Muller (2013). The core argument in the proof is nevertheless the same, that is an application of the bipolar theorem but with an originality, the introduction of the notion of Hilbert basis. Notice that a closely related result - equivalence between statements (a) and (b) - can be found in Proposition 3.4, Page 237 in Marshall (1991), in the continuous case.

Our discrete framework is justified by two reasons. First, even variables which are often assumed continuous in theory (such as income), are provided on a discrete scale in empirical data (euro cents, for instance). Then, this framework is particularly

${ }^{10}$ As illustrated in Section 8, one notices that such a result is not true when $\succeq_{\mathcal{T}}$ is not a discrete (or convex) cone. That confirms, again, that the definition of the sequence of transfers as a cone is a crucial assumption in our framework. 
appropriate for ordinal variables (health, education, ... ), which are usually defined on an ordered categorical scale. This last point will be detailed in Section 6 .

Without loss of generality, we consider that the set of transfers $\mathcal{T}$ is minimal in the sense of Definition 3. Minimality of a set - in this sense - is not hard to establish, thus it is just a matter of adequate definition for $\mathcal{T}$. What follows is our main result.

Theorem 2. Let $\mathcal{T}$ be a minimal set of transfers. For all $m, m^{\prime} \in \mathbb{Z}^{|\mathcal{S}|}$ such that $\sum_{s \in \mathcal{S}} m_{s}=\sum_{s \in \mathcal{S}} m_{s}^{\prime}<\infty$, the following three statements are equivalent:

(a) $m \succeq_{\mathcal{T}} m^{\prime}$,

(b) $W(m) \geq W\left(m^{\prime}\right), \forall W \in \mathcal{W}_{\mathcal{T}}$,

(c) $W_{u}(m) \geq W_{u}\left(m^{\prime}\right), \forall u \in \mathcal{U}_{\mathcal{T}}$.

Proof. The equivalence $(a) \Leftrightarrow(b)$ is proved in Proposition 2. The proof of the implication $(a) \Rightarrow(c)$ is, actually, equivalent to the proof of $(b) \Rightarrow(a)$ in Theorem 1.

It remains to prove $(c) \Rightarrow(a)$. Let $m, m^{\prime} \in \mathbb{Z}^{|\mathcal{S}|}$ such that $\sum_{s \in \mathcal{S}} m_{s}=\sum_{s \in \mathcal{S}} m_{s}^{\prime}<\infty$, and assume that (c) is true. Moreover, let $b(m, u)=-\sum_{s \in \mathcal{S}} m_{s} u_{s}$. The polar cone of $\mathcal{U}_{\mathcal{T}} \subset \mathcal{U}$, under the duality $(\mathcal{M}, \mathcal{U} ; b)$, is defined by:

$$
\mathcal{U}_{\mathcal{T}}^{\circ}=\left\{m \in \mathcal{M} \mid b(m, u) \leq 0, \forall u \in \mathcal{U}_{\mathcal{T}}\right\}
$$

By definition, $\left(m-m^{\prime}\right) \in \mathcal{M}$. From statement (c), we also have $b\left(m-m^{\prime}, u\right)=$ $-\sum_{s \in \mathcal{S}}\left(m_{s}-m_{s}^{\prime}\right) u_{s} \leq 0$, whenever $u \in \mathcal{U}_{\mathcal{T}}$. It follows that $\left(m-m^{\prime}\right) \in \mathcal{U}_{\mathcal{T}}^{\circ}$. Now the polar cone of $\mathcal{T} \subset \mathcal{M}$, under the duality $(\mathcal{M}, \mathcal{U} ; b)$, is defined by:

$$
\mathcal{T}^{\circ}=\{u \in \mathcal{U} \mid b(m, u) \leq 0, \forall m \in \mathcal{T}\} .
$$

From definition of $\mathcal{U}_{\mathcal{T}}$ in $(9)$ and $b\left(m-m^{\prime}, u\right)$, one immediately observes that $\mathcal{U}_{\mathcal{T}}=\mathcal{T}^{\circ}$. Because $\left(m-m^{\prime}\right) \in \mathcal{U}_{\mathcal{T}}^{\circ}$ and $\mathcal{U}_{\mathcal{T}}=\mathcal{T}^{\circ}$, it follows that $\left(m-m^{\prime}\right) \in \mathcal{T}^{\circ \circ}$, where $\mathcal{T}^{\circ \circ}$ is the bipolar cone of $\mathcal{T}$.

We then recall that $(\mathcal{M}, \mathcal{U} ; b)$ is a strict dual pair (Lemma 4$)$. By applying the bipolar theorem (Lemma 3), we know that $\mathcal{T}^{\circ \circ}$ is the smallest closed set containing the convex cone generated by $\mathcal{T}$, which can be written as $\mathcal{D}_{o}(\mathcal{T})=\operatorname{co}\left\{\lambda m \mid \lambda \in \mathbb{R}_{+}, m \in \mathcal{T}\right\}$. Notice that, as $\mathcal{T}$ is a discrete and finite set, $\mathcal{D}_{o}(\mathcal{T})$ is closed. Moreover $\mathcal{T} \subset \mathbb{Z}^{|\mathcal{S}|}$. As integers are rational numbers, $\mathcal{D}_{o}(\mathcal{T})$ is also a rational cone. By applying Lemma 1 , we know that $\mathcal{D}_{o}(\mathcal{T})$ is generated by a (unique) minimal Hilbert basis. Because $\mathcal{T}$ is here defined as a minimal set of transfers (according to Definition 3 ), we have $\mathscr{H}\left(\mathcal{D}_{o}(\mathcal{T})\right)=\mathcal{T}$. Then, because $\left(m-m^{\prime}\right) \in \mathcal{T}^{\circ \circ}=\mathcal{D}_{o}(\mathcal{T})$ but also $\left(m-m^{\prime}\right) \in \mathbb{Z}^{|\mathcal{S}|}$, one concludes that $\left(m-m^{\prime}\right) \in \mathcal{D}_{o}(\mathcal{T}) \cap \mathbb{Z}^{|\mathcal{S}|}$. By definition of an Hilbert basis, such $\left(m-m^{\prime}\right)$ can be expressed as positive linear combination of elements in this basis, with coefficients in $\mathbb{Z}_{+}$. one deduces that $\left(m-m^{\prime}\right) \in \mathcal{D}(\mathcal{T})$, or equivalently $m \succeq_{\mathcal{T}} m^{\prime}$.

To sum up, if the sequence of transfers we are interested in can be described as a binary relation $\succeq_{\mathcal{T}}$ induced by a discrete cone - as assumed in this paper, and which 
is actually the case for most of the transfers considered in the literature - then the previous theorem guarantees that the unanimity of ranking among all the social welfare functions consistent with $\succeq_{\mathcal{T}}\left(\mathcal{W}_{\mathcal{T}}\right.$ in the general case, or $\mathcal{U}_{\mathcal{T}}$ if we restrict attention to the utilitarian subclass) is a necessary and sufficient condition to reach dominance according to $\succeq_{\mathcal{T}}$.

\section{Application to a unidimensional ordinal variable}

The discrete framework developed in this paper is useful to investigate cardinal variables, even those usually treated in theory as continuous variables. The main example is income which is always defined, in practice, on a finitely divisible scale (euro cents, for instance). More importantly, this discrete framework is required for ordinal variables defined on an ordered categorical scale, which is the case for most of the dimensions characterizing individual's social welfare, aside for income (for instance variables such as self-reported health or happiness, access to basic services, or educational achievements).

The question of inequality and social welfare measurement for ordered categorical variables is investigated, among others, by Gravel et al. (2014). ${ }^{11}$ Their objective is to provide a result comparable to the Hardy et al. (1952) theorem (denoted hereafter HLP), initially developed for a cardinal and continuous variable. Consider as an example health, self-reported on a scale with categories 'very bad', 'bad', 'so-so', 'good' and 'very good', and assume that this variable is interpersonally comparable. One the one hand, we can say that social welfare experienced by an individual is higher in one category rather than another one, lower in the scale. On the other hand it is quite impossible to provide, with compelling arguments, an unambiguous measure of the magnitude of social welfare one applies to each category and, even more troublesome, of the distance in social welfare between two categories. Gravel et al. (2014) take the route that, for such a variable, the ranking of the categories is the only relevant information to assess social welfare. In that case, we face a purely ordinal variable the scale is defined up to an increasing transformation - which cannot be, by definition, 'summed' or 'averaged' across the individuals.

Consider an outcome scale $\mathcal{S}=\{1,2, \ldots, K\}$ defined up to an increasing transformation, where $K \geq 3$ is fixed ( $K$ is equal to 5 in the previous self-reported health example). A distribution is a list $n=\left(n_{1}, \ldots, n_{K}\right) \in \mathcal{N}$, with $\mathcal{N}=\left\{n \in \mathbb{Z}_{+}^{K} \mid \sum_{s \in \mathcal{S}} n_{s}=\right.$ $N\}$. The equalizing transfer proposed by Gravel et al. (2014) refers to the equity principle introduced by Hammond (1976) in social choice theory: A distribution $n$ is obtained from distribution $n^{\prime}$ by mean of a (weak) Hammond transfer if $n=n^{\prime}$, or if there exist $i, j, k, l \in \mathcal{S}$ such that $i<j \leq k<l$ and:

$$
\begin{gathered}
n_{s}=n_{s}^{\prime}, \text { for all } s \in \mathcal{S} \backslash\{i, j, k, l\}, \\
\left(n_{i}-n_{i}^{\prime}\right)=\left(n_{l}-n_{l}^{\prime}\right)=-1, \\
\left(n_{j}-n_{j}^{\prime}\right)=\left(n_{k}-n_{k}^{\prime}\right)=1 \text { if } j \neq k, \quad \text { and } \quad\left(n_{j}-n_{j}^{\prime}\right)=2 \text { if } j=k .
\end{gathered}
$$

${ }^{11}$ An extension for ordinal but continuous variables is developed in Gravel et al. (2019). 
The authors argue that the notion of Hammond transfer is the only relevant way to define an equalizing process in an ordinal setting. As the scale is defined up to an increasing transformation, the equalizing process has to be invariant to such transformation. This condition is satisfied by Hammond transfers, but violated by the standard Pigou-Dalton progressive income transfer (which is well-suited to a cardinal variable, see Section 8). They also establish the necessary and sufficient condition to be apply on the utilitarian class of social welfare functions to ensure consistency with Hammond transfers. Precisely, they obtain the following result.

Proposition 3 (Gravel et al. (2014), Prop. 4, Page 15). The following two statements are equivalent:

(a) For all $n, n^{\prime} \in \mathcal{N}$, $n$ being obtained from $n^{\prime}$ by means of a finite sequence of (arbitrary) Hammond transfers implies $W_{u}(n) \geq W_{u}\left(n^{\prime}\right)$,

(b) $u \in\left\{\left(u_{1}, \ldots, u_{K}\right) \in \mathbb{R}^{K} \mid\left(u_{j}-u_{i}\right) \geq\left(u_{l}-u_{k}\right), \forall i<j \leq k<l\right\}$.

The methodology developed in this paper is well-suited to complete the previous result in order to obtain, without any other proof, the equivalence between dominance according to a sequence of Hammond transfers and dominance according to the utilitarian class of social welfare functions consistent with these transfers. First of all, as we have defined a general set of transfers $\mathcal{T}$ in Definition 1, we define below the set $\mathcal{T}_{H}$ of Hammond transfers:

Definition 5 (Set of Hammond transfers). The set $\mathcal{T}_{H}$ of (weak) Hammond transfers is the set of all $m \in \mathbb{Z}^{|\mathcal{S}|}$ defined as follows. Either $m=0$, or there exist $i, j, k, l \in \mathcal{S}$ such that $i<j \leq k<l$ and:

$$
\begin{gathered}
m_{s}=0, \text { for all } s \in \mathcal{S} \backslash\{i, j, k, l\} \\
m_{i}=m_{l}=-1, \\
m_{j}=m_{k}=1 \text { if } j \neq k \text {, and } m_{j}=2 \text { if } j=k .
\end{gathered}
$$

As in Definition 2, we can formally define the notion of sequence of transfers in $\mathcal{T}_{H}$. In that case, for all $m, m^{\prime} \in \mathbb{Z}^{|\mathcal{S}|}$, we write $m \succeq_{\mathcal{T}_{H}} m^{\prime}$ if and only if $\succeq_{\mathcal{T}_{H}}$ is induced by the discrete cone generated by $\mathcal{T}_{H}$.

It is not difficult to verify that $\mathcal{T}_{H} \subset \mathcal{T}$. Indeed, $\mathcal{T}_{H}$ satisfies all the properties of a set of transfers as described in Definition 1, among of them the independence requirement (see Remark 1). Then, let $\mathcal{W}_{\mathcal{T}_{H}}$ be the set of social welfare functions consistent with $\succeq_{\mathcal{T}_{H}}$ (see Definition 4). One also observes from our Theorem 1 that the class $\mathcal{U}_{\mathcal{T}_{H}}$, defined in Equation (9) but with $\mathcal{T}$ replaced by $\mathcal{T}_{H}$, is equivalent to the one defined in Statement (b), Proposition 3. Finally one remarks that the transfers in $\mathcal{T}_{H}$ are minimal, in the sense of Definition 3. It follows that Theorem 2, with $\mathcal{T}_{H}$ instead of the more general set $\mathcal{T}$, also applies in that context. One obtains the following result: 
Corollary 1. For all $n, n^{\prime} \in \mathcal{N}$, the following three statements are equivalent:

(a) $n \succeq_{\mathcal{T}_{H}} n^{\prime}$,

(b) $W(n) \geq W\left(n^{\prime}\right), \forall W \in \mathcal{W}_{\mathcal{T}_{H}}$,

(c) $W_{u}(n) \geq W_{u}\left(n^{\prime}\right), \forall u \in \mathcal{U}_{\mathcal{T}_{H}}$.

This equivalence is helpful to complete the HLP theorem for ordered categorical variables, as wanted by Gravel et al. (2014).

\section{Application to a bidimensional variable}

In order to illustrate that the set of possible applications of our model is large, we consider as second example the case of a bidimensional variable, consisting of a first dimension which is cardinally measurable and transferable between individuals, and a second dimension which is assumed ordinal and non-transferable (respectively income and health, for instance). This framework is investigated by Gravel and Moyes (2012). We assume here that both dimensions take a finite number of values (euro cents for income), ${ }^{12}$ such that an outcome is a dot $s=(i, j) \in \mathbb{Z}_{+}^{2}$, with $0 \leq i \leq I$ and $0 \leq j \leq H$, with $I$ and $H$ fixed. The set of outcomes is thus a finite and fixed set $\mathcal{S} \subset \mathbb{Z}_{+}^{2}$. As in the general framework (see Section 3), we denote by $n=\left(n_{s}\right)_{s \in \mathcal{S}} \in \mathcal{N}$ a distribution, recalling that the population size is fixed, namely $\sum_{s \in \mathcal{S}} n_{s}=N$. Gravel and Moyes (2012) consider two inequality-reducing transfers, as described below.

They first introduce the notion of favorable income permutation, which consists in exchanging the income of an individual with that of another individual, initially worse-off in both dimensions. Consider for instance an initial distribution $n^{\prime}$ and two individuals, the first (the worse-off) with outcome $(i, j)$ and the second (the bestoff) with outcome $(k, l)$, such that $(i, j)<(k, l)$. After permutation, one obtains distribution $n$ such that outcomes of all are not changed, apart for the worse-off (of the two individuals mentioned above) who reaches outcome $(k, j)$ and the best-off who, in return, obtains $(i, l)$. An illustration is provided in Figure 3.a. Now we define the set of favorable income permutations.

Definition 6 (Set of favorable income permutations). The set $\mathcal{T}_{1}$ of (weak) favorable income permutations is the set of all $m \in \mathbb{Z}^{|\mathcal{S}|}$ defined as follows. Either $m=0$, or there exist $i, j, k, l$ with $0 \leq i, k \leq I$ and $0 \leq j, l \leq H$ and $(i, j) \ll(k, l)$ such that:

$$
\begin{gathered}
m_{s}=0, \text { for all } s \in \mathcal{S} \backslash\{(i, j),(k, l)\}, \\
m_{(i, j)}=m_{(k, l)}=-1, \text { and } m_{(i, l)}=m_{(k, j)}=1 .
\end{gathered}
$$

${ }^{12}$ As emphasized by the authors themself (Gravel and Moyes, 2012, Page 1392), the fact that both dimensions take a finite number of values is not a restriction, even if they assume in their paper that the cardinally measurable variable (income) is continuous. 
a. A favorable income permutation

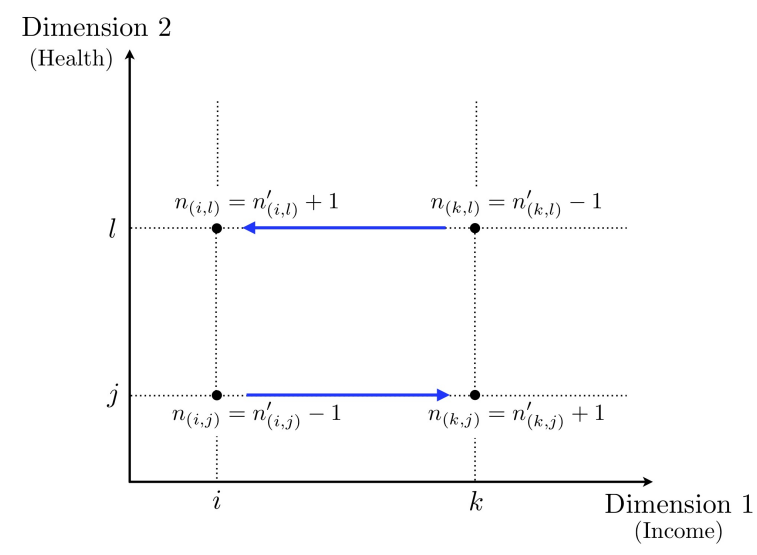

b. A within-type progressive income transfer

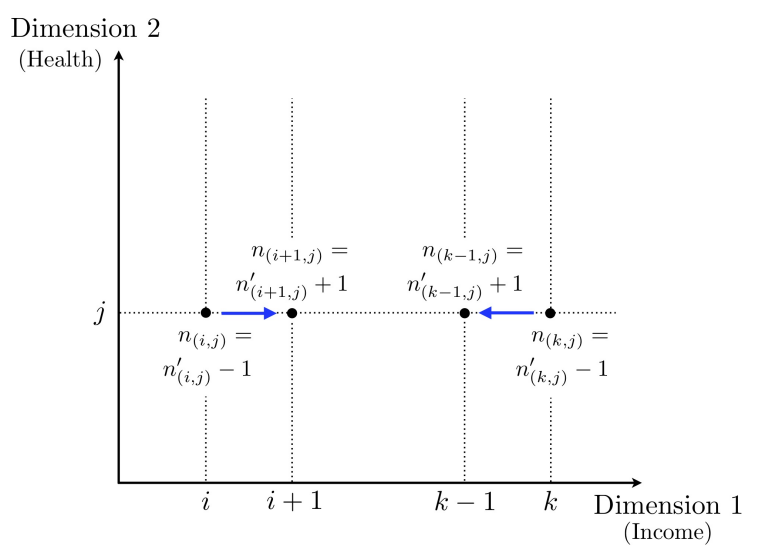

Figure 3: Examples of bidimensional welfare-improving transfers

Then, Gravel and Moyes (2012) discuss the notion of within-type progressive income transfer, which consists in a progressive income transfer between two individuals having the same health status. Consider an initial distribution $n^{\prime}$ and two individuals, the first (the worse-off) with outcome $(i, j)$ and the second (the best-off) with outcome $(k, j)$, such that $i<k$. After transfer one obtains distribution $n$ such that their outcomes are, respectively $(i+1, j)$ and $(k-1, j)$, without any modification for the individuals not concerned by the transfer. This transfer is illustrates in Figure 3.b.

Definition 7 (Set of within-type progressive income transfers). The set $\mathcal{T}_{2}$ of (weak) within-type progressive income transfers is the set of all $m \in \mathbb{Z}^{|\mathcal{S}|}$ defined as follows. Either $m=0$, or there exist $0 \leq i<k \leq I$ and $0 \leq j \leq H$, such that:

$$
\begin{gathered}
m_{s}=0, \text { for all } s \in \mathcal{S} \backslash\{(i, j),(i+1, j),(k-1, j),(k, j)\}, \\
\qquad m_{(i, j)}=m_{(k, j)}=-1, \\
m_{(i+1, j)}=m_{(k-1, j)}=1 \text { if } i+1 \neq k-1, \text { and } m_{(i+1, j)}=2 \text { if } i+1=k-1 .
\end{gathered}
$$

Because it is assumed that both favorable income permutations and within-type progressive income transfers are inequality-reducing transfers, it could be interesting to pool them together into a larger set of 'admissible' transfers. For instance, one could directly investigate the set $\mathcal{T}_{3}=\mathcal{T}_{1} \cup \mathcal{T}_{2}$. For each of these three types of transfers permutation, income transfer or the combination of both - Gravel and Moyes (2012) obtain a result comparable to Proposition 3 in the previous Section (Lemmas 4.1, 5.1 and 5.2 in their paper). On the basis of these results, one deduces that the class of utilitarian social welfare functions consistent with transfers in $\theta=\mathcal{T}_{1}, \mathcal{T}_{2}, \mathcal{T}_{3}$, characterized by the sets $\mathcal{U}_{\theta}$ as defined in Equation (9) can be written as, respectively:

$$
\begin{gathered}
\mathcal{U}_{\mathcal{T}_{1}}=\left\{u \in \mathcal{U} \mid u_{(i+1, j)}-u_{(i, j)} \geq u_{(i+1, l)}-u_{(i, l)}, \forall i, \forall j<l\right\}, \\
\mathcal{U}_{\mathcal{T}_{2}}=\left\{u \in \mathcal{U} \mid u_{(i+1, j)}-u_{(i, j)} \geq u_{(k+1, j)}-u_{(k, j)}, \forall i<k, \forall j\right\}, \\
\mathcal{U}_{\mathcal{T}_{3}}=\mathcal{U}_{\mathcal{T}_{1}} \cap \mathcal{U}_{\mathcal{T}_{2}} .
\end{gathered}
$$


Then, sequences of transfers in $\theta=\mathcal{T}_{1}, \mathcal{T}_{2}, \mathcal{T}_{3}$ can be written through the quasiordering $\succeq_{\theta}$ (see Definition 2) such that for all $m, m^{\prime} \in \mathbb{Z}^{|\mathcal{S}|}$, we write $m \succeq_{\theta} m^{\prime}$ if and only if $\succeq_{\theta}$ is induced by the discrete cone generated by $\theta$. We also let $\mathcal{W}_{\theta}$ be the set of social welfare functions consistent with $\succeq_{\theta}$ (see Definition 4). Because $\theta \subset \mathcal{T}$ and because $\theta$ is minimal in the sense of Definition 3 (for $\theta=\mathcal{T}_{1}, \mathcal{T}_{2}, \mathcal{T}_{3}$ ) our Theorem 2, with $\theta$ instead of the more general set $\mathcal{T}$, directly applies. One obtains:

Corollary 2. Let $\theta=\mathcal{T}_{1}, \mathcal{T}_{2}, \mathcal{T}_{3}$. For all $n, n^{\prime} \in \mathcal{N}$, the following three statements are equivalent:

(a) $n \succeq_{\theta} n^{\prime}$,

(b) $W(n) \geq W\left(n^{\prime}\right), \forall W \in \mathcal{W}_{\theta}$,

(c) $W_{u}(n) \geq W_{u}\left(n^{\prime}\right), \forall u \in \mathcal{U}_{\theta}$.

The results for $\theta=\mathcal{T}_{1}, \mathcal{T}_{3}$ are also established in Gravel and Moyes (2012) - respectively in Theorems 4.1 and 5.1 - but with the need of other proof arguments. Our general methodology based one discrete cones ensures, immediately, that the results are true.

\section{Social deprivation: transfers outside the framework}

Some sets of transfers considered in the literature are not consistent with our model. In this section, we provide an example investigated by Chateauneuf and Moyes (2006) and Magdalou and Moyes (2009), which deals with equalization processes for (unidimensional) income distributions. The framework is equivalent to the one described in Section 6. We consider a set of possibles (euro cent) incomes $\mathcal{S}=\{1,2, \ldots, K\}$ with a fixed $K \geq 3$, and distributions written as lists $n=\left(n_{1}, \ldots, n_{K}\right) \in \mathcal{N}$, with $\mathcal{N}=\left\{n \in \mathbb{Z}_{+}^{K} \mid \sum_{s \in \mathcal{S}} n_{s}=N\right\}$. The difference from Section 6 relates to the interpretation of $\mathcal{S}$. Because income is a cardinal variable, the distance between two incomes is relevant for measurement. It follows that we can compute, for instance, the mean income. For a distribution $n \in \mathcal{N}$, it is equal to $\mu(n)=\sum_{s \in \mathcal{S}} n_{s} s$.

We first introduce the notion of Pigou-Dalton progressive income transfer $(P T)$. We say that distribution $n$ is obtained from distribution $n^{\prime}$ by mean of a (weak) progressive income transfer if $n=n^{\prime}$, or if there exist $i, l \in \mathcal{S}$ such that $i<l$ and:

$$
\begin{gathered}
n_{s}=n_{s}^{\prime}, \text { for all } s \in \mathcal{S} \backslash\{i, i+1, l-1, l\}, \\
\left(n_{i}-n_{i}^{\prime}\right)=\left(n_{l}-n_{l}^{\prime}\right)=-1, \\
\left(n_{i+1}-n_{i+1}^{\prime}\right)=\left(n_{l-1}-n_{l-1}^{\prime}\right)=1 \text { if } i+1 \neq l-1, \text { and }\left(n_{i+1}-n_{i+1}^{\prime}\right)=2 \text { if } i+1=l-1 .
\end{gathered}
$$

This is a particular case of Hammond transfer (as described in Section 6), where in addition the increase (on the income scale) of individual having income $i$ is equal to the decrease of individual having income $l$. In other terms, this is an Hammond transfer with an equal mean restriction, such that $\mu(n)=\mu\left(n^{\prime}\right)$. 
As emphasized in Chateauneuf and Moyes (2006) and Magdalou and Moyes (2009), a number of experimental studies have established that the inequality-reducing impact of a progressive transfer is not unanimously accepted. ${ }^{13}$ Based on the theory of social deprivation (Runciman, 1966), they propose alternative principle of transfers which impose solidarity among the individuals involved in the equalizing process. We present here one example, called uniform of the right progressive income transfer (URPT). According to this principle, if some income is taken from an individual, the same amount has to be taken from every individual who is as rich or richer than him. Then the total amount collected is transferred to a poorer individual. This is therefore a (mean-preserving) progressive income transfer, with solidarity among the richer individuals.

Proposition 4 (Chateauneuf and Moyes (2006)). Let $\theta=$ PT, URPT. The following two statements are equivalent: ${ }^{14}$

(a) For all $n, n^{\prime} \in \mathcal{N}$, $n$ being obtained from $n^{\prime}$ by means of a finite sequence of transfers $\theta$ implies $W_{u}(n) \geq W_{u}\left(n^{\prime}\right)$,

(b) $u \in\left\{\left(u_{1}, \ldots, u_{K}\right) \in \mathbb{R}^{K} \mid\left(u_{i+1}-u_{i}\right) \geq\left(u_{l+1}-u_{l}\right), \forall i<l\right\}$.

The previous result highlights the lack of flexibility of the utilitarian model to distinguish the inequality views captured by PT and URPT transfers. In both cases, the condition to be imposed on the utility is concavity (Statement (b)). Our model sheds new light on this surprising result, as discussed below. We first introduce the set of PT transfers.

Definition 8 (Set of PT transfers). The set $\mathcal{T}_{P T}$ of (weak) PT transfers is the set of all $m \in \mathbb{Z}^{|\mathcal{S}|}$ defined as follows. Either $m=0$, or there exist $i, l \in \mathcal{S}$ such that $i+1<l$ and:

$$
\begin{gathered}
m_{s}=0, \text { for all } s \in \mathcal{S} \backslash\{i, i+1, l-1, l\} \\
\qquad m_{i}=m_{l}=-1, \\
m_{i+1}=m_{j-1}=1 \text { if } i+1 \neq j-1, \quad \text { and } \quad m_{i+1}=2 \text { if } i+1=j-1 .
\end{gathered}
$$

One immediately observes that $\mathcal{T}_{P T} \subset \mathcal{T}_{H}$, with $\mathcal{T}_{H}$ the set of Hammond transfers defined in Section 6. As $\mathcal{T}_{H} \subset \mathcal{T}$, our model perfectly fits for sequences of $P T$ transfers. It follows that Corollary 1 still applies, by substituting $\mathcal{T}_{P T}$ for $\mathcal{T}_{H}$. One deduces from Proposition 4 that the set $\mathcal{U}_{\mathcal{T}_{P T}}$ of utilities consistent with transfers in $\mathcal{T}_{P T}$ (as defined in Equation (9)) is equivalent to the set described in Statement (b) of the same Proposition, namely the set of concave utilities. Because Proposition 4 also concerns URPT transfers, a question arises: Can one obtain an equivalent result for

\footnotetext{
${ }^{13}$ Amiel and Cowell (1992) is the ground-breaking experimental study in this field.

${ }^{14}$ This result is not directly proved in Chateauneuf and Moyes (2006). It results from Propositions 1, 3 and 5, respectively in Pages 28, 43 and 52 .
} 
these transfers? The answer is no, as expected. ${ }^{15}$ We demonstrate that a sequence of URPT transfers cannot be described as a discrete cone, through a simple example.

Consider an income scale $\mathcal{S}=\{1,2,3,4\}$ and a distribution $n^{\prime}=(1,1,1,0)$, for a society consisting of 3 individuals. Assume that one unit of income is taken from the individual having initially 3 units, and given to the individual having initially 1 unit. This transformation is a URPT transfer and we obtain, after transfer, distribution $n=(0,3,0,0)$. If the set of $U R P T$ transfers is a subset of $\mathcal{T}$ (Definition 1 ) we know from Remark 1 that, if $n, n^{\prime} \in \mathcal{N}$ and $n$ is obtained from $n^{\prime}$ by means of a URPT transfer, then $m=n+\epsilon$ is also obtained from $m=n+\epsilon$ by means of a URPT transfer (as soon as $\epsilon$ is such that $\left.m, m^{\prime} \in \mathcal{N}\right)$. Consider the admissible $\epsilon=(0,-1,0,1)$, so that $m=(1,0,1,1)$ and $m^{\prime}=(0,2,0,1)$. Obvioulsy $m$ cannot be obtained from $m^{\prime}$ by means of a URPT transfer, or a sequence of such transfers. As the independence requirement described in Remark 1 is not satisfied, the set of URPT transfers cannot be a subset of $\mathcal{T}$. It follows that a sequence of such transfers cannot be written as a discrete cone, in line with Definition 2.

As presented in Section 5, our model puts forward the close relationship between sequences of transfers which can be written as discrete cones, and the utilitarian class of social welfare functions. The inability of a sequence of URPT transfers to be written as a discrete cone explains why this class of social welfare functions is not well-suited to capture the inequality views of such transfers.

\section{Extension to decision-making under risk: the key role of the EU model}

The appropriate way to represent individual preferences in situations of decisionmaking under risk remains an important research issue. The expected utility model has, for many reasons (among of them its simplicity and tractability) a prominent place in the literature. But at the same time, some of the underlying assumptions are not consistent with behavior observed in practice such that, for instance, the independence axiom. Since Machina (1982), a large literature puts forward the key role of the expected utility model for modeling risk attitudes, even for non-expected utility preferences. In this Section we provide new arguments in this way.

A (unidimensional) lottery is usually represented by a cumulative distribution, denoted $F$ or $G$, which is defined on a closed interval $I$ of $\mathbb{R}$. The standard approach consists in assuming an expected utility representation of the decision maker's preferences. In that case, letting $u$ be a utility function defined on $I$ and continuous, $F$ is considered as weakly better than $G$ if and only if $\int_{I} u(x) d F(x) \geq \int_{I} u(x) d G(x)$. The unanimity of ranking within a class of utility functions, usually called an (integral) stochastic order, is often used to distinguish distributions in terms of risk. For instance, usually, $F$ is considered less risky than $G$ when $G$ is a mean-preserving spread of $F$. And it

${ }^{15}$ Indeed, a URPT transfer can be obtained by means of a finite sequence of PT transfers, but the reverse is wrong. Hence, if the set $\succeq_{\mathcal{T}_{U R P T}}$ would exist, we would have $\succeq_{\mathcal{T}_{U R P T}} \subset \succeq_{\mathcal{T}_{P T}}$ and dominance according to the class of utilitarian social welfare function with concave utilities, cannot be equivalent to dominance according to $\succeq_{\mathcal{T}_{P T}}$ and, at the same time, dominance according to $\succeq_{\mathcal{T}_{U R P T}}$. Only the first equivalence is true, as well-known in the literature. 
is well-known that, for any distributions $F$ and $G$ such that $\int_{I} x d F(x)=\int_{I} x d G(x)$, $G$ is a mean-preserving spread of $F$ if and only if $\int_{I} u(x) d F(x) \geq \int_{I} u(x) d G(x)$ for all $u$ weakly concave.

A first question is how non-expected utility preferences can be adressed. By assuming that the decision maker's preferences can be represented by a continuous and smooth functional - that is a (Gâteaux) differentiable functional $V$ which associates to each cumulative distribution a value in $\mathbb{R}$ - Machina (1982) has established that any non-expected utility decision maker acts locally as an expected utility maximizer. This argument is based on the following observation:

$$
V(G)-V(F) \approx \int_{I} v_{F}(x) d G(x)-\int_{I} v_{F}(x) d F(x),
$$

where $v_{F}$ is the derivative of $V$ at $F$, a continuous function. This derivative is usually called local utility, as the difference $V(G)-V(F)$ can be approximated by the difference in the expected value of $v_{F}$ with respect to $G$ and $F$. We emphasize that this observation relies on the differentiability assumption required for $V$.

A second issue deals with the consistency of non-expected utility preferences with stochastic orders, as these criteria are usually related to transparent notions of risk reduction, through simple probability mass transfers (such as mean-preserving spreads). By considering again a continuous and smooth functional $V$ representing the decision maker's preferences, Cerreia-Vioglio et al. (2016) establish the following general result.

Proposition 5 (Cerreia-Vioglio et al. (2016), Prop. 1, Page 1102). Let $\mathcal{U}^{\star}$ be a set of bounded and continuous functions on $I$. The following two statements are equivalent:

(a) For all distributions $F$ and $G$ defined on $I, \int_{I} u(x) d F(x) \geq \int_{I} u(x) d G(x)$ for all $u \in \mathcal{U}^{\star}$ implies $V(F) \geq V(G)$,

(b) The set of all derivatives of $V$ (namely, all the local utilities) is included in the closed convex cone generated by $\mathcal{U}^{\star}$ and all the constant functions.

This result establishes the necessary and sufficient condition to be placed on the functional $V$, to make this preferences representation consistent with the stochastic order characterized by the set of utilities $\mathcal{U}^{\star}$.

As established by Muller (2013) most of the stochastic orders, even for multivariate distributions, can be associated to sequence of probability mass transfers which can be written as convex cones. On this basis, we can provide some answers to the two questions previously raised. We first slightly modify our framework. We still consider a partially ordered, finite and fixed set of outcomes $\mathcal{S} \subset \mathbb{Z}_{+}^{d}$ where each dimension is, for instance, monetary variables written in euro cents. A (probability) distribution is a list of $p=\left(p_{s}\right)_{s \in \mathcal{S}}$, where $p_{s}$ indicates the probability of having ( $d$-dimensional) outcome $s \in \mathcal{S}$, and $\mathcal{P}=\left\{p \in \mathbb{R}_{+}^{|\mathcal{S}|} \mid \sum_{s \in \mathcal{S}} p_{s}=1\right\}$ indicates the set of distributions. The set $\mathcal{T}$ of (probability mass) transfers, assumed to be compact, is the set of all $m \in \mathbb{R}^{|\mathcal{S}|}$ which can be written as $m=p-p^{\prime}$ for some $p, p^{\prime} \in \mathcal{P}$, and that $m \in \mathcal{T}$ and $(-m) \in \mathcal{T}$ imply $m=0$. A sequence of transfers is described by the relation $\succeq_{\mathcal{T}}$, 
such that we write $p \succeq_{\mathcal{T}} p^{\prime}$ if and only if $\succeq_{\mathcal{T}}$ is induced by the convex cone generated by $\mathcal{T}$. The decision maker's preferences are represented by the function $W: \mathcal{P} \rightarrow \mathbb{R}$. Finally let $\mathcal{U}_{\mathcal{T}}$ be a set of utility vectors $u=\left(u_{s}\right)_{s \in \mathcal{S}} \in \mathbb{R}^{|\mathcal{S}|}$ consistent with $\succeq_{\mathcal{T}}$, and let $W_{u}(p)=\sum_{s \in \mathcal{S}} p_{s} u_{s}$.

Adopting this revisited framework our Theorem 2, applied to probability distributions $p, p^{\prime} \in \mathcal{P}$, remains true. The proof arguments to establish the equivalence between statements in (a) and (b) (presented in Proposition 2) are still valid, and the equivalence between (a) and (c) results from Theorem 2.4.1 in Muller (2013). This result provide an answer - partial and in a specific context, of course - to the first question raised above, namely how to represent non-expected utility preferences. If the objective is to reach a unanimity of ranking between two probability distributions, for any decision-makers - even with non-expected utility preferences - who agree with the risk-reducing impact of transfers in $\mathcal{T}$ (mean-preserving spread, for instance), it is is sufficient to check the unanimity among the expected utility maximizers. This observation puts forward the core aspect of expected utility preferences, in a situation where a definition of 'risk reduction' is unanimously approved. Notice that we are in a multivariate realm, and that no 'smoothness' assumptions are required for the decision maker's preferences.

In order to complement Proposition 5, we have the following result. It is based on our Theorem 2 and is almost equivalent to Theorem 2 in Marshall et al. (1967). We emphasize that, again, we deal with multivariate variables, without any smoothness assumption.

Proposition 6. The following two statements are equivalent:

(a) For all $p, p^{\prime} \in \mathcal{P}, W_{u}(p) \geq W_{u}\left(p^{\prime}\right)$ for all $\mathcal{U}_{\mathcal{T}}$ implies $W(p) \geq W\left(p^{\prime}\right)$,

(b) $W(p+\lambda m)-W(p) \geq 0$ for all $p \in \mathcal{P}$, and all $m \in \mathcal{T}, \lambda>0$ such that $p+\lambda m \in \mathcal{P}$.

Proof (sketch of). $(a) \Rightarrow(b)$. Let $p \in \mathcal{P}$, and $m \in \mathcal{T}, \lambda>0$ such that $p+\lambda m \in \mathcal{P}$. By definition we have $(p+\lambda m) \succeq_{\mathcal{T}} p$ or equivalently, from Theorem $2, W_{u}(p+\lambda m) \geq$ $W_{u}(p)$ for all $\mathcal{U}_{\mathcal{T}}$. Now assume that (a) is true. It follows that $W(p+\lambda m)-W(p) \geq 0$.

$(b) \Rightarrow(a)$. Assume that (b) is true and that $W_{u}(p) \geq W_{u}\left(p^{\prime}\right)$ for all $\mathcal{U}_{\mathcal{T}}$, or equivalently $p \succeq_{\mathcal{T}} p^{\prime}$ (from Theorem 2). We then use the same arguments as in the proof of Theorem 2 in Marshall et al. (1967), which can be sketched as follows. Because $p \succeq_{\mathcal{T}} p^{\prime}$, there exists $T$ such that we can write $p-p^{\prime}=\sum_{t=1}^{T} \lambda_{t} m_{\cdot t}$ with $\lambda_{t}>0$ and $m_{\cdot t} \in \mathcal{T}$. From statement (b) and transitivity of $W$, and because $p=p^{\prime}+\sum_{t=1}^{T} \lambda_{t} m_{\cdot t}$, we obtain $W(p)-W\left(p^{\prime}\right) \geq 0$.

This result is restricted to stochastic orders which can be associated to sequence of mass transfers written as convex cones. In practice, this restriction is not so demanding (see Muller, 2013). It establishes the necessary and sufficient condition to be placed on $W$, to make this preferences representation consistent with such a stochastic order. Statement (b) can be interpreted - loosely speaking because $W$ is not here assumed differentiable - to a set of restrictions on the directional derivatives of $W$. It is thus a simple, and helpful remark to answer the second question raised above, namely how to characterize consistency of non-expected preferences with stochastic orders. 


\section{Conclusion}

In this paper, we present a general model for assessing social welfare within a discrete and multidimensional framework. On the basis of a normative definition of what can be called a 'social welfare improvement', we present a result in the vein of the Hardy et al. (1952)'s theorem, establishing the equivalence between three quasi-orderings. The first is a dominance criterion based on a sequence of such transfers, reflecting our normative views on social welfare. The second is an unanimous ranking of distributions among the utilitarian social planners who agree with these welfare views, whereas the last quasi-ordering extends this unanimity to the largest class of social welfare functions sharing these views.

This result, provided at an abstract level, can be applied in various context. It can be used for uni- or multi-dimensional variables, with cardinal or ordinal dimensions. The only requirement is that the considered set of transfers can be written as a discrete cone. For instance, it can be used to complete the HLP-type theorem for a discrete ordinal variable, partially obtained in Gravel et al. (2014). It is worth noting that, due to its additive structure, the utilitarian class of social welfare functions is closely related to the sequence of transfers we consider, defined as a quasi-ordering induced by a discrete cone. The utilitarian class consistent with the welfare views provided by the transfers appears to be a core set of preferences, as the unanimity of ranking within this class is necessary but also sufficient to reach the unanimity in the largest class of social welfare functions (also consistent with these views).

Whereas most of the transfers studied in the literature fall within this framework, others do not. This is the case of the progressive transfers with solidarity among the individuals involved in the equalizing process (Chateauneuf and Moyes, 2006; Magdalou and Moyes, 2009), which can not be written as discrete cones, unlike the Pigou-Dalton progressive transfers. This particular feature explains why the utilitarian class cannot distinguish these transfers to the standard Pigou-Dalton ones.

This paper provides a generalization of the equivalence between the two first statements of the Hardy et al. (1952)'s theorem. An interesting extension could be to investigate a direct equivalence with the third statement, namely an empirically implementable criterion which is, in practice, the most relevant tool to compare distributions on real data. This question might be the object of future researches.

\section{References}

Amiel, Y. and Cowell, F. (1992). Measurement of income inequality: Experimental test by questionnaire. Journal of Public Economics, 47, 3-26.

Atkinson, A. (1970). On the measurement of inequality. Journal of Economic Theory, 2, 244-263.

Cerreia-Vioglio, S., Maccheroni, F. and Marinacci, M. (2016). Stochastic dominance analysis without the independence axiom. Management Science, 63, 1097-1109. 
Chateauneuf, A. and Moyes, P. (2006). A non-welfarist approach to inequality measurement. In M. McGillivray (ed.), Inequality, Poverty and Well-Being, Hampshire/New-York: Palgrave Macmillan, Basingtoke, pp. 22-65.

Dasgupta, P., Sen, A. K. and Starrett, D. (1973). Notes on the measurement of inequality. Journal of Economic Theory, 6, 180-187.

Fields, G. and FEI, J. (1978). On inequality comparisons. Econometrica, 46, 305316.

Gordan, P. (1873). Ueber die auflösung linearer gleichungen mit reellen coefficienten. Mathematische Annalen, 6, 23-28.

Gravel, N., Magdalou, B. and Moyes, P. (2014). Ranking distributions of an ordinal attribute, AMSE Working Paper 1450, Aix-Marseille School of Economics.

- - - and - (2019). Inequality measurement with an ordinal and continuous variable. Social Choice and Welfare, forthcoming.

- and Moyes, P. (2012). Ethically robust comparisons of bidimensional distributions with an ordinal attribute. Journal of Economic Theory, 147, 1384-1426.

HAmmond, P. J. (1976). Equity, arrow's conditions and rawls's difference principle. Econometrica, 44, 793-803.

Hardy, G. H., Littlewood, J. E. and Polya, G. (1952). Inequalities, 2nd edition. Cambridge, UK: Cambridge University Press.

Hilbert, D. (1890). Uber die theorie der algebraischen formen. Mat. Ann., 36, 473534.

Kolm, S.-C. (1969). The optimal production of social justice. In J. Margolis and H. Guitton (eds.), Public Economics, London: Macmillan, pp. 145-200.

Machina, M. (1982). "expected utility" analysis without the independence axiom. Econometrica, 50, 277-323.

Magdalou, B. and Moyes, P. (2009). Deprivation, welfare and inequality. Social Choice and Welfare, 32, 253-273.

Marshall, A. (1991). Multivariate stochastic orderings and generating cones of functions. In K. Mosler and M. Scarsini (eds.), IMS Lecture Notes Monograph Series 19, Hayward, CA: Inst. Math. Stat.

-, Olkin, I. and ARnold, B. (2011). Inequalities: Theory of Majorization and its Applications. New-York: Springer, Springer Series in Statistics, Second Edition.

-, Walkup, D. and Wets, R.-B. (1967). Order-preserving functions: Applications to majorization and order statistics. Pacific Journal of Mathematics, 23, 569-584.

Muller, A. (2013). Duality theory and transfers for stochastic order relations. In H. Li and X. Li (eds.), Sochastic Orders in Reliability and Risk, New York: Lectures Notes in Statistics 208, Springer, pp. 137-155.

- and Scarsini, M. (2012). Fear of loss, inframodularity, and transfers. Journal of Economic Theory, 147, 1490-1500.

Rockafellar, R. (1970). Convex Analysis. Princeton: Princeton University Press.

Runciman, W. (1966). Relative Deprivation and Social Justice. London: Routledge and Kegan Paul.

SchriJver, A. (1986). Theory of Linear and Integer Programming. Chichester: Wiley-Interscience.

SEn, A. (1973). On Economic Inequality. Oxford: Clarendon Press.

VAN DER CORPUT, J. (1931a). Konstruktion der minimalbasis für spezielle diophan- 
A MODEL OF SOCIAL WELFARE IMPROVING TRANSFERS

tische systeme von linear-homogenen gleichungen und ungleichungen. Proceedings Koninklijke Akademie van Wetenschappen te Amsterdam, 34, 515-523.

(1931b). Ueber diophantische systeme von linear-homogenen gleichungen und ungleichungen. Proceedings Koninklijke Akademie van Wetenschappen te Amsterdam, 34, 372-382. 


\section{CEE-M Working Papers' - 2018}

WP 2018 - 01: $\quad$ Ilaria Brunetti., Mabbel Tidball, $\&$ Denis Couvet « Relationship Between Biodiversity and Agricultural Production »

WP 2018 - 02: $\quad$ Phillippe Le Coent, Raphaële Préget \& Sophie Thoyer « Do farmers follow the herd? The influence of social norms in the participation to agri-environmental schemes.»

WP 2018 - 03: $\quad$ Ludivine Roussey \& Raphaël Soubeyran

« Overburdened judges »

WP 2018 - 04: $\quad$ Nicolas Quérou

«Interacting collective action problems in the Commons »

WP 2018 - 05: $\quad$ Karine Constant \& Marion Davin

«Unequal vulnerability to climate change and the transmission of adverse effects through international trade »

WP 2018 - 06: $\quad$ Henrik Andersson \& Emmanuelle Lavaine

« Nitrates and property values: evidence from a french market intervention »

WP 2018 - 07: $\quad$ Mamadou Gueye, Nicolas Querou \& Raphaël Soubeyran

«Does equity induce inefficiency? An experiment on coordination »

WP 2018 - 08: $\quad$ Douadia Bougherara \& Laurent Piet

« On the role of probability weighting on WTP for crop insurance with and without yield skewness»

WP 2018 - 09: $\quad$ Douadia Bougherara, Carole Ropars-Collet \& Jude Saint-Gilles

« Impact of private labels and information campaigns on organic and fair trade food demand»

WP 2018 - 10: $\quad$ Sylvain Chabé-Ferret, Philippe Le Coent, Arnaud Reynaud, Julie Subervie $\&$ Daniel Lepercq

« Can we nudge farmers Into saving water? Evidence from a randomized experiment »

WP 2018 - 11: $\quad$ Dimitri Dubois, Stefano Farolfi, Phu Nguyen-Van \& Juliette Rouchier "Information sharing is not always the right option when it comes to CPR extraction management: experimental finding»

WP 2018 - 12: $\quad$ Tristan Le Cotty, Elodie Maitre d'Hotel, Raphaël Soubeyran \& Julie Subervie

« Inventory credit as a commitment device to save grain until the hunger season»

${ }^{1}$ CEE-M Working Papers

Contact : laurent.garnier@inra.fr/ 0499613121 
WP 2018 - 13:

Brice Magdalou

"A model of social welfare improving transfers »

WP 2018 - 14:

Mickael Beaud, Mathieu Lefebvre $\&$ Julie Rosaz

« Other-regarding preferences and giving decision in risky environments: experimental evidence » 